\title{
Strain-induced superconductor-insulator transition on a Lieb lattice
}

\author{
Nyayabanta Swain $\odot^{1, *}$ and Madhuparna Karmakar $\circledast^{2, \dagger}$ \\ ${ }^{1}$ School of Physical and Mathematical Sciences, Nanyang Technological University, 21 Nanyang Link, Singapore 637371 \\ ${ }^{2}$ Department of Physics, Indian Institute of technology, Madras, Chennai-600036, India
}

(Received 17 December 2019; revised manuscript received 9 March 2020; accepted 6 April 2020; published 7 May 2020)

\begin{abstract}
We report the numerical investigation of strain-induced superconductor-insulator quantum phase transition on a Lieb lattice. Based on a nonperturbative Monte Carlo technique, which retains the spatial fluctuations of the superconducting pairing field at all orders but neglects the temporal fluctuations, we show that in two dimensions, an $s$-wave superconductor undergoes transition to a highly correlated bosonic insulator under the influence of strain, applied as staggered hopping amplitudes. We further demonstrate a strain-induced BCS-BEC like crossover in the superconducting state, such that the superconductor-insulator transition takes place between a bosonic superconductor and a bosonic insulator. Our results suggest that it is the contribution of the dispersive bands towards the superconducting order that dictates this crossover. To the best of our knowledge, this is the first work to report a theoretical investigation of "disorder free" superconductor-insulator phase transition in systems with Lieb lattice structure. With the recent experimental realization of the Lieb lattice in ultracold atomic gases, photonic lattices as well as in solid state systems, we believe that the results presented in this paper would be of importance to initiate experimental investigation of such novel quantum phase transitions. We further discuss the fate of such systems at finite temperature, highlighting the effect of fluctuations on the superconducting pair formations, thermal scales, and quasiparticle behavior. Our nonperturbative numerical approach to the problem enables us to capture the thermal scales of the system accurately and provides us with mean-field estimates of the ground state properties. The high-temperature quasiparticle signatures discussed in this paper are expected to serve as benchmarks for experiments such as radio frequency and momentum resolved radio frequency spectroscopy measurements carried out on systems such as ultracold atomic gases.
\end{abstract}

DOI: 10.1103/PhysRevResearch.2.023136

\section{INTRODUCTION}

Tuning the quantum behavior of a material by applying force via external strain has been in the forefront of research in condensed matter systems, over the past few years [1-7]. A novel way of altering the lattice structure has always been chemical doping which exerts chemical pressure on the lattice. The technique however often demands for stringent experimental conditions. An alternate way to manipulate the lattice geometry of materials is via strain engineering. Extensive experimental works, fuelled by the need of designing quantum devices and materials, carried out on strain engineering over the past few years have shown that the technique is capable of giving rise to exotic quantum phases and phase transitions [8-10].

It has been demonstrated recently that by applying tensile strain to $\mathrm{LaCoO}_{3}$ films a strain-induced high-temperature ferromagnetic insulator could be realized which opens up

\footnotetext{
*nyayabanta@ntu.edu.sg

†madhuparna.k@gmail.com

Published by the American Physical Society under the terms of the Creative Commons Attribution 4.0 International license. Further distribution of this work must maintain attribution to the author(s) and the published article's title, journal citation, and DOI.
}

possibility of future devices with high operation temperatures [1]. Strain-induced competition between charge order and interfacial superconductivity (with $T_{c}$ as high as $\sim 8.3 \mathrm{~K}$ ) has been observed in $\mathrm{SnSe}_{2}$ films grown on $\mathrm{SrTiO}_{3}$ substrates [2]. This observation suggests the possibility of engineering twodimensional (2D) materials in which strong strain and charge injection can enhance or even induce superconductivity [2]. Furthermore, strain has been found to enhance the superconducting $T_{c}$ upto a factor of two in $\mathrm{SrTiO}_{3}$ films [3]. In the same spirit, strain-induced superconductivity with $T_{c} \sim 10 \mathrm{~K}$ has been reported for $\mathrm{BaFe}_{2} \mathrm{As}_{2}$ [4]. Recently, anisotropic in-plane strains were applied to oxygen octahedral sublattice of $\mathrm{VO}_{2}$ and an intriguing behavior of in-plane orientation dependent metal-insulator transition was reported [5]. Lastly, strain engineering of graphene is now an well established area of research with exciting promises $[6,7,11,12]$. The cumulative outcome of these experimental observations put forward strain as a new and promising tuning parameter to control quantum many body properties.

Yet another forerunner of modern condensed matter physics are the "designer lattices" [13]. These are artificial lattice structures which can be engineered in ultracold atomic gases, photonic crystals or even in solid state systems. The lattice parameters can be tuned via external agencies so as to achieve the desired effect. One such category of designer lattices are the flat band lattices, characterized by one or more dispersionless spectral bands. The kinetic energy in such 
bands are quenched and the single-particle spectra is independent of momentum, giving rise to the flat bands [14-16]. While the theoretical possibility of lattices with flat bands was known since three decades [17-21], it was only recently that they received the renewed and much deserved interest, owing to the proposals of optical lattice experiments to realize designer lattices. One of the simplest flat band lattice in two dimension is a Lieb lattice, basically a depleted square lattice with three sites unit cell. The dispersion spectra of the same comprises of three spectral bands with two of them being dispersive and one flat, centered at the Fermi level. The noninteracting band structure consists of a single Dirac cone at the corners of the first Brillouin zone, intersected by the flat band [22].

Over the past couple of years Lieb lattice has been successfully engineered under different experimental settings viz. optical Lieb lattice in bosonic cold atoms [23,24], designer two-dimensional materials in which artificial lattices are engineered through lithography and atomic manipulations [25-27], optically induced photonic Lieb lattices [28-30], etc. These experimental realizations of the Lieb lattice has indeed opened up a Pandora's box of future possibilities both in terms of experimental and theoretical investigations.

We take cue from the two experimental advancements discussed above, viz. (i) strain engineering and (ii) realization of designer lattices, and attempt to understand the behavior of a quantum many body system on a flat band Lieb lattice, being subjected to strain. It is well known that interaction between quantum particles in flat bands can lead to spontaneous symmetry breaking and emergence of correlated quantum states [31-36]. The simplest model which takes into account the effect of interaction between the lattice fermions is the Hubbard model and over the past couple of years substantial effort has been invested to analyze the physics of Hubbard model on flat band Lieb lattice, based on the mean-field theory (MFT) [37-40] as well as other numerically sophisticated techniques such as, dynamical mean-field theory (DMFT) [41-43], determinant quantum Monte Carlo (DQMC), etc. $[36,44]$. Based on the DMFT and DQMC studies, the fate of the repulsive Hubbard model on the Lieb lattice is now relatively well established both at the ground state and at finite temperature [41,44]. Regarding the attractive Hubbard model, theoretical investigations are being carried out to capture the behavior of a superconducting state on the Lieb lattice. Recently MFT has been used to map out the ground-state phase diagram of population imbalanced fermionic superfluid on the Lieb lattice [37]. Furthermore, using MFT and DMFT calculations, the attractive Hubbard model on a Lieb lattice with staggered hopping has been studied in detail so as to understand the contribution of the flat band to superfluid weight in this lattice $[38,45]$.

Attempts made to access the finite temperature physics of such a system within the purview of MFT, would understandably overestimate the thermal scales. On the other hand, DMFT though gives a better estimate of the thermal scales as compared to the MFT, fails to capture the spatial fluctuations correctly due to its single unit cell approach. Away from the weak-coupling limit, spatial fluctuations are expected to be strong and provides additional thermal scales to the system. Moreover, as we demonstrate in this work,

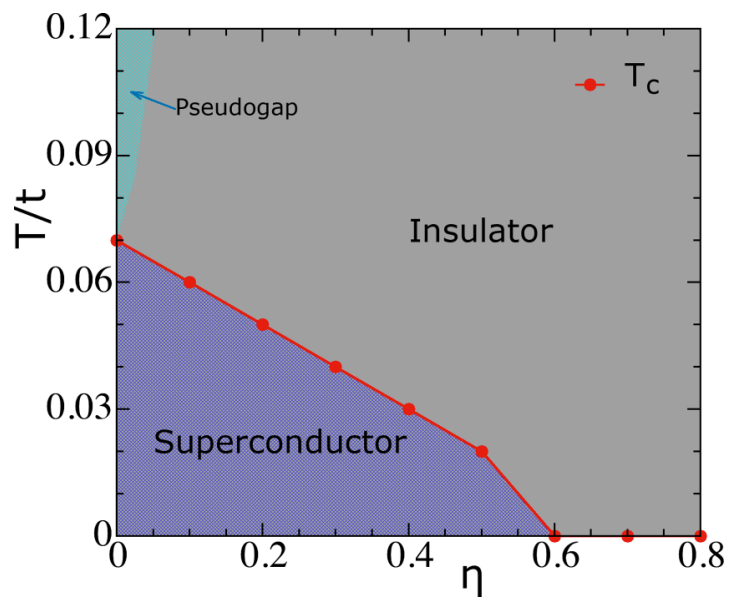

FIG. 1. Strain-temperature $(\eta-T)$ phase diagram showing the superconductor-insulator transition (SIT) in our model. The quantum phase transition occurs at a critical strain of $\eta_{c}=0.6$ for $U=2 t$. The various phases are (i) a gapped superconductor, (ii) a bosonic insulator, and (iii) a pseudogap phase. The red (dotted) curve corresponds to the $T_{c}$ of the system.

interaction significantly renormalizes the behavior of the superconducting order even at the ground state and a single unit cell approach to the problem is insufficient to capture such renormalizations. This leaves a gap in our understanding of the physics of superconductors on a flat band Lieb lattice, even for an unstrained or isotropic system, and demands for a theoretical investigation to address these issues.

The interplay between strongly correlated quantum state on a flat band lattice and applied strain presents one with an interesting premise to understand the localization-delocalization transitions. It must be noted that one of the salient features of the flat band lattices such as the Lieb lattice is strong localization of energy, giving rise to "compact localized states" $[13,46]$. In the noninteracting limit, these states are immune to strain, and unlike the curious case of graphene [6,7], strain (as applied through staggered hopping amplitudes) does not lead to a gap opening at the Fermi level. On the other hand, interactions between the quantum particles open up a gap at the Fermi level of the flat band Lieb lattice and as mentioned above, give rise to symmetry breaking quantum states, such as a superconducting state. The question we ask in this paper is how the application of strain affects this superconducting state and what are the phase transitions that it promotes?

Figure 1 constitutes the principal result of this paper where we demonstrate a strain $(\eta)$ induced quantum phase transition between a superconductor and an insulator at a fixed interaction strength, on a Lieb lattice. The transition is determined via a Monte Carlo technique which retains the spatial fluctuations of the superconducting pairing field at all orders but ignores the temporal fluctuations, thus differing from DQMC method. However, it is complimentary to DMFT. The quantum phase transition discussed in this paper is thus a mean-field estimate of the same. While we do not expect any qualitative change in the low temperature results discussed in this paper, inclusion of quantum fluctuations might lead to quantitative changes in the estimates of the phase boundaries. To the best of our knowledge this is the first theoretical 
proposal to realize a disorder free superconductor-insulator transition (SIT) on the flat band Lieb lattice. Consequently, our work is expected to initiate experimental investigations of the same in the artificial Lieb lattices, which are now an experimental reality. While we discuss our formalism and the results obtained from the same in the following sections we highlight our main observations here. (i) In the absence of strain, the system undergoes the BCS-BEC crossover as a function of increasing interaction $U$. The maximum $T_{c}$ is achieved for $U=U_{c} \sim 4 t$, (ii) At a selected $U$, strain induces a quantum SIT. Superconductivity is lost beyond a critical strain, through the loss of long-range phase coherence. Notably, application of strain alters the superconducting state from BCS-like to BEC-like, even at weak coupling, i.e., there is a strain-induced BCS-BEC crossover. (iii) The system is "bosonic" on either side of the transition, i.e., a BEC-like superconducting state and a bosonic insulator, respectively. The single-particle spectral gap remains hard across the phase transition. (iv) Strain dramatically alters the quasiparticle spectral behavior. While in the unstrained limit the dispersion spectra is characterized by a single flat and two dispersive bands, the spectra at the strong strain limit comprises of three (gapped) flat bands, at and away from the Fermi level, where the energy states are strongly localized.

The rest of the paper is organized as follows, we discuss the numerical technique used to carry out this work in Sec. II, along with the superconducting and quasiparticle indicators based on which our results are analyzed. The results presented in Sec. III comprises of two parts. In the first part, we set the stage by analyzing the unstrained superconducting system on the Lieb lattice. The second part is dedicated to investigating the strain-induced SIT at a particular $U$, which constitutes the focus area of this work. We discuss the relevance of our work from the perspective of experiments in Sec. IV and touch upon certain aspects of the numerical technique used in this work. This is followed by the conclusions drawn based on this work.

\section{MODEL AND METHOD}

We define our superconducting system through a twodimensional attractive Hubbard model on a Lieb lattice as [38]

$$
\begin{aligned}
H= & -\sum_{\langle i j\rangle, \sigma} t_{i j}\left(c_{i, \sigma}^{\dagger} c_{j, \sigma}+\text { H.c. }\right) \\
& -|U| \sum_{i} \hat{n}_{i, \uparrow} \hat{n}_{i, \downarrow}+\mu \sum_{i, \sigma} \hat{n}_{i, \sigma},
\end{aligned}
$$

where $t_{i j}=(1 \pm \eta) t$ is the staggered hopping amplitude as shown in Fig. 2, for the nearest neighbors and is zero otherwise. $t=1$ sets the energy scale of the problem. The strain is introduced in the system in terms of staggered hopping, through the parameter $\eta$. The unit cell of Lieb lattice comprises of three sites marked in red, blue and green, in Fig. 2. In each unit cell the red and green constitutes the bond sites (being on the $x$ and $y$ bonds of a square lattice plaquette) while the blue corresponds to the rim sites (the sites corresponding to the square lattice plaquette). The strain $(\eta)$ is introduced in a way such that increasing $\eta$ leads to larger hopping amplitude between the intracell sites, while simultaneously

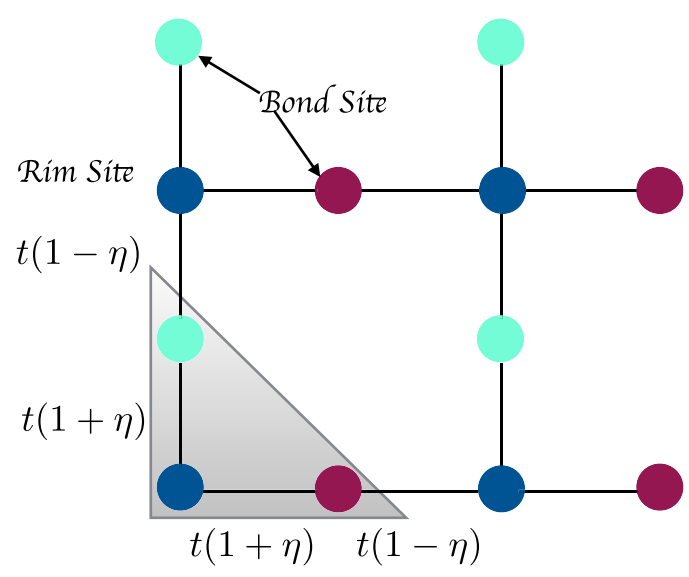

FIG. 2. Schematic diagram showing the structure of Lieb lattice. The three site unit cell structure of the lattice is highlighted. The sites in blue are called the rim sites, while the one in red and green are the bond sites along the $x$ and $y$ directions, respectively. The magnitude of strain is given by $\eta$ and it is applied as staggered hopping.

reducing the hopping amplitude between the neighboring unit cells. We choose to work in a grand canonical ensemble and thus at a fixed chemical potential $\mu ;|U|>0$ is the attractive interaction between the fermions.

We decompose the four fermion term in the pairing channel using Hubbard-Stratonovich (HS) decomposition and introduce a "bosonic" auxiliary complex scalar field $\Delta_{i}(\tau)=$ $\left|\Delta_{i}(\tau)\right| e^{i \theta_{i}(\tau)}$, where $\left|\Delta_{i}(\tau)\right|$ correspond to the amplitude and $\theta_{i}(\tau)$ the phase of the superconducting pairing field. In principle, the interaction can be decomposed in charge channel as well. However, the inclusion of a charge field along with the pairing would simply shift the Fermi level of the system without leading to any qualitative change in our results. We have verified that in spite of working in the grand canonical ensemble the number density of fermions do not drift significantly from its $T=0$ value (see Appendix). At the same time, a single channel decomposition of the interaction cuts down the computation cost significantly and thereby allows us to access sufficiently large system sizes, to capture spatial inhomogeneties. We thus restrict ourselves to the single channel decomposition and allow for two bosonic auxiliary fields $\left|\Delta_{i}(\tau)\right|$ and $\theta_{i}(\tau)$. We consider $s$-wave symmetric pairing field. For $|U|>t$, a mean-field description of the system breaks down and one needs to retain the fluctuations beyond the mean field. The auxiliary field comprising of both spatial and temporal degrees of freedom can be numerically treated exactly through DQMC, but is restricted to smaller system sizes (specially since the unit cell of a Lieb lattice is thrice that of an ordinary square lattice).

We use an alternative static path approximation (SPA) [47-50] technique to address the problem wherein we retain all the spatial fluctuations (and not just the saddle point fluctuations) of the auxiliary fields but drop the temporal fluctuations. The system can thus be envisioned as free fermions moving in a random background of "classical" $\Delta_{i}$. The resulting effective Hamiltonian thus takes the 
form

$$
\begin{aligned}
H_{\text {eff }}= & -t(1 \pm \eta) \sum_{\langle i j\rangle, \sigma}\left(c_{i, \sigma}^{\dagger} c_{j, \sigma}+\text { H.c. }\right)+\mu \sum_{i, \sigma} \hat{n}_{i, \sigma} \\
& +\sum_{i}\left(\Delta_{i} c_{i, \uparrow}^{\dagger} c_{i, \downarrow}^{\dagger}+\Delta_{i}^{*} c_{i, \downarrow} c_{i, \uparrow}\right)+\sum_{i} \frac{\left|\Delta_{i}\right|^{2}}{|U|}
\end{aligned}
$$

where the last term corresponds to the stiffness cost associated with the auxiliary field. This numerical technique is akin to the MFT at $T=0$, and becomes progressively more accurate as $T \rightarrow \infty$, capturing the thermal scales accurately. The pairing field configurations follow the probability distribution,

$$
P\left\{\Delta_{i}\right\} \propto \operatorname{Tr}_{c, c^{\dagger}} e^{-\beta H_{\mathrm{eff}}},
$$

where $\beta$ is the inverse temperature. This is related to the free energy of the fermions. For large and random $\left\{\Delta_{i}\right\}$, the fermion trace is computed numerically, the corresponding configurations are generated via classical Monte Carlo technique, diagonalizing $H_{\text {eff }}$ for each attempted update of $\left\{\Delta_{i}\right\}$. The relevant fermionic correlators are then computed on the optimized configurations at different temperatures. This numerically expensive technique scales with the system size as $O\left(N^{4}\right)$, where $N=3 L^{2}$ is the number of lattice sites. The computational cost has been cut down by using traveling cluster approximation (TCA), wherein instead of diagonalizing the entire lattice for each attempted update, we diagonalize a smaller cluster centered around the update site [49]. Both SPA and TCA has been extensively bench marked for several quantum many body problems [49-53] and the results obtained are found to be in excellent quantitative agreement with those obtained by DQMC.

The results presented in this paper corresponds to a system size of $N=768$ sites with the cluster size being $N_{c}=48$ sites. We have also verified our results with $N_{c}=108$ and have found them to be robust with respect to the size of the cluster. Finite system size analysis (upto $N=1200$ ) has been carried out and the results presented in this paper are found to be immune to system size changes. The parameter space encompassed by $\{\mu,|U|, \eta, T\}$ is huge and we restrict ourselves over relevant cross sections in this parameter space. We set the chemical potential to $\mu=-0.2 t$ (corresponding to a fermionic number density of $n \approx 0.9$ ), such that the system is not at but close to half filling. Owing to the $\mathrm{SO}(3)$ symmetry of the Hubbard model, at half filling the ground state of the system comprises of degenerate superconducting and charge density wave orders. We have selected the filling to be away from this point of degeneracy such that the stable ground state is a superconductor. This allows us to avoid the charge density wave fluctuations and justifies our approximation of single channel decomposition of the Hubbard interaction. For the BCS-BEC crossover, the interaction regime of $t \leqslant|U|<10 t$ has been explored, while the effect of strain is reported on a selected interaction cross section of $U=2 t$, for $\eta \in[0: 1]$. We have verified that our results are qualitatively immune to the choice of the interactions, Our ground-state calculations are carried out at $T=0.01 t$ corresponding to one hundredth of the hopping scale and is verified of their robustness upto $T=0.001 t$. We analyze our results based on the following superconducting and quasiparticle indicators:

(1) distribution of the pairing field amplitude: $P(|\Delta|)=$ $\left\langle\sum_{i} \delta\left(|\Delta|-\left|\Delta_{i}\right|\right)\right\rangle$;

(2) average phase correlation of pairing field: $\frac{1}{N}\left\langle\sum_{i, j} \cos \left(\theta_{i}-\theta_{j}\right)\right\rangle$;

(3) real-space maps: (a) pairing field amplitude $\left|\Delta_{i}\right|$ and (b) pairing field phase correlation $\cos \left(\theta_{0}-\theta_{i}\right)$;

(4) single-particle density of states (DOS): $N(\omega)=$ $\left\langle\frac{1}{N} \sum_{i, n}\left(\left|u_{n}^{i}\right|^{2} \delta\left(\omega-E_{n}\right)+\left|v_{n}^{i}\right|^{2} \delta\left(\omega+E_{n}\right)\right)\right\rangle$;

(5) spectral function and line shapes: $A(\mathbf{k}, \omega)=$ $-(1 / \pi) \operatorname{Im} G(\mathbf{k}, \omega)$.

Here, $\quad G(\mathbf{k}, \omega)=\left.\lim _{\delta \rightarrow 0} G\left(\mathbf{k}, i \omega_{n}\right)\right|_{i \omega_{n} \rightarrow \omega+i \delta}, \quad$ where $G\left(\mathbf{k}, i \omega_{n}\right)$ is the imaginary frequency transform of $\left\langle c_{\mathbf{k}}(\tau)\right.$ $\left.c_{\mathbf{k}}^{\dagger}(0)\right\rangle . u_{n}^{i}$ and $v_{n}^{i}$ are the Bogoliubov de-Gennes (BdG) eigenvectors corresponding to the eigenvalues $E_{n}$ for the configuration under consideration.

\section{RESULTS}

We now go back to the main observation of this work, i.e., strain-induced SIT, shown in Fig. 1. We note that the system undergoes a SIT at a critical strain of $\eta_{c} \sim 0.6$ for an interaction strength of $U=2 t$. The high-temperature pseudogap regime is restricted at $\eta \rightarrow 0$, suggesting that strain renders the spectral gap at the Fermi level immune to thermal fluctuations. Application of strain strongly suppresses the superconducting transition temperature $\left(T_{c}\right)$ of the system. The observation is obvious, as progressively increasing strain (as applied in our model) decouples the three-site unit cells from each other.

In order to analyze the different phases shown in this phase diagram, one needs to understand the "unstrained" system based on the indicators mentioned above. We thus discuss the BCS-BEC crossover on the Lieb lattice in the following few sections and then focus on a specific interaction strength of this crossover regime to demonstrate the effect of strain, in the later sections of the paper.

\section{A. BCS-BEC crossover}

In Fig. 3, we present the thermal phase diagram of the BCS-BEC crossover on an unstrained Lieb lattice. Note that the thermal transitions discussed in this paper are BerezinskyKosterlitz-Thouless (BKT) transitions corresponding to the algebraic decay of long-range order in two dimensions. The interaction-temperature $(U-T)$ phase diagram shown in Fig. 3 comprises of four key thermodynamic phases viz. (a) superconductor, (b) metal, (c) insulator, and (d) pseudogap. The phases are demarcated by two thermal scales corresponding to the loss of (quasi) long-range superconducting phase coherence at $T_{c}$ and the loss of short-range superconducting pair correlations at $T_{p g}$. The behavior of $T_{c}$ with increasing $U$ is nonmonotonic, with the maximum $\left(T_{c} \sim 0.12 t\right)$ at $U \sim 4 t$, corresponding to the unitarity, in the context of ultracold atomic gases [54]. Similar nonmonotonicity has been observed in the behavior of the superfluid weight as a function of interaction [38]. Based on superfluid weight calculations, at half filling the maximum $T_{c}$ has been found to be $\sim 0.13 t$ at an interaction strength of $U \sim 3.5 t$. The observation is in excellent agreement with the one presented in Fig. 3 for the 


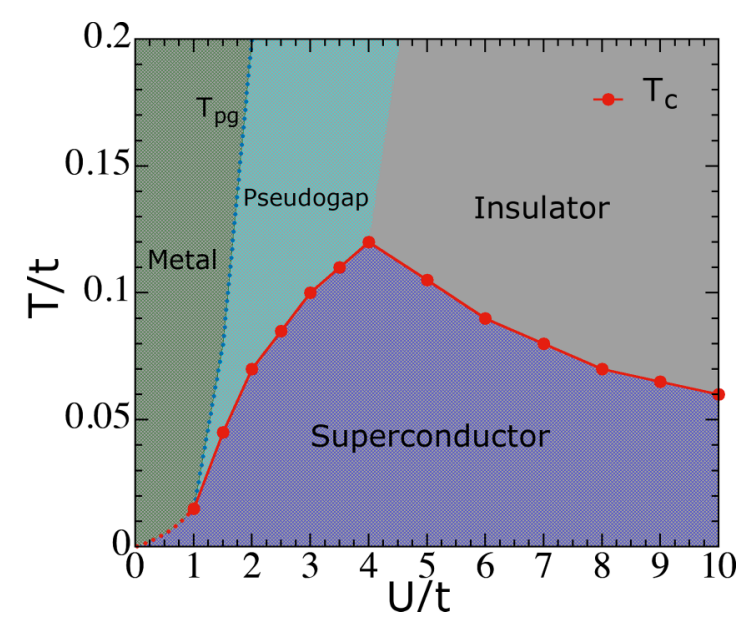

FIG. 3. Thermal phase diagram showing the BCS-BEC crossover on the unstrained Lieb lattice. The behavior of $T_{c}$ is nonmonotonic with the peak at $U \sim 4 t$. The ground state shows a gapped $s$-wave superconducting state. At the finite temperature, the weak interaction regime correspond to a metallic state, while the strong interaction regime is an insulator. The intermediate interaction regime correspond to pseudogap phase characterized by short-range pair correlations

system close to half filling. We note that while at $T<T_{c}$ any finite interaction gives rise to a superconducting order, the high-temperature $T>T_{c}$ phases pertaining to different interaction regimes are significantly different.

For $U \leqslant t$, superconductivity emerges from a hightemperature metallic Fermi liquid phase. In this regime $T_{c} \sim$ $T_{p g}$, suggesting that the loss of short-range superconducting pair correlations and long-range phase coherence are almost simultaneous. In this weak-coupling regime, the loss of superconducting order is dictated by the suppression of pairing field amplitude and the thermal scale obeys the relation $T_{c} \sim U$.

Over the intermediate regime of interaction $t \leqslant U \leqslant 4 t$, the low temperature superconducting order emerges from a nontrivial strongly correlated high-temperature phase. We mark this phase as the pseudogap in Fig. 3 and demonstrate that $T_{p g} \gg T_{c}$ in this regime, indicating the survival of shortrange pair correlations upto temperatures significantly higher than the one corresponding to the loss of phase coherence. The loss of superconductivity in this regime is governed by phase fluctuations and requires a nonperturbative treatment, to get captured. Since spatial fluctuations are dominant at high temperatures, a single unit cell approach such as DMFT and its variants are inadequate to capture the behavior of the system in this regime.

In the strong-coupling regime of $U>4 t$, superconductivity emerges from a high-temperature gapped phase akin to a correlated bosonic insulator. The phase is characterized by large amplitudes of the pairing field but a vanishing phase correlation and the thermal scale behaves as $T_{c} \sim t^{2} / U$. Here we note that based on DQMC calculations it was predicted that $T_{c}$ scale is strongly suppressed in the BEC regime on a Lieb lattice $\left(T_{c} \sim 0.03 t\right.$ at $\left.U=8 t\right)$, as compared to its square lattice counterpart [55]. Our analysis however shows a fairly robust superconducting order in the strong-coupling regime on the Lieb lattice, with $T_{c} \sim 0.08 t$ at $U=8 t$. We emphasize that this discrepancy is due to strong finite size effect arising out of the small system size on which DQMC calculations were carried out (see Appendix B). Compared to the $N \sim 100$ used for the DQMC calculations, the results presented in this paper corresponds to a system size of $N=768$.

\section{Global thermodynamic indicators}

Next, we show the global indicators based on which the thermodynamic phases are demarcated in the phase diagram. In Fig. 4(a), the thermal evolution of the average superconducting phase correlation $\left[\left\langle\cos \left(\theta_{i}-\theta_{j}\right)\right\rangle\right]$ is presented for different $U$, where, $\theta_{i}$ and $\theta_{j}$ correspond to the phases of the pairing field at lattice sites $i$ and $j$, respectively. The point of inflection of each curve corresponds to the $T_{c}$, at that $U$. The figure shows the nonmonotonic evolution of $T_{c}$ with respect to $U$, with the peak $T_{c} \sim 0.12 t$ being at $U \sim 4 t$. The thermal evolution of the average pairing field amplitude is shown next, in Fig. 4(b), normalized by the corresponding values at $T=0$. While the amplitude expectedly increases with $U$, the interesting observation is that $\left\langle\left|\Delta_{i}\right|\right\rangle \neq 0$ even when $T \gg$ $T_{c}$. The behavior is in remarkable contrast to the mean-field theory which suggests $\left\langle\left|\Delta_{i}\right|\right\rangle=0$ at $T \geqslant T_{c}$. Figures 4(a) and 4(b) together shows the impact of thermal fluctuations on the system, away from the weak coupling. While thermal fluctuations destroy long-range phase coherence at $T_{c}$, shortrange pair correlations survive even at $T \gg T_{c}$, leading to a nonzero $\left\langle\left|\Delta_{i}\right|\right\rangle$. Finally, Fig. 4(c) shows the superconducting gap versus $T_{c}$ ratio as a function of increasing interaction. In the BCS limit, this ratio is 3.5. As shown in Fig. 4(c), at $U=2 t$, the ratio $\sim 18$, which is significantly above the BCS prediction and grows as $\sim(U / t)^{2}$ at large $U$. The figure demonstrates that the $T=0$ gap is not a suitable indicator of the robustness of the superconducting state, beyond the weak-coupling regime.

In order to highlight the effect of thermal fluctuations on the pairing field, we next show the distribution of the pairing field amplitude at different temperatures for selected interactions representative of the (i) weak $(U=2 t)$ [we select $U=2 t$ for the weak-coupling limit since for $U \lesssim t$ the correlation length $(\xi)$ of the superconducting pairs become comparable to our system size], (ii) intermediate $(U=4 t)$, and (iii) strong $(U=6 t)$ coupling regimes, in Fig. 5. For any interaction, at the lowest temperature the amplitude of the pairing field $\left|\Delta_{i}\right|$ exhibits a narrow distribution (ideally a delta function), with the mean corresponding to the $T=0$ mean-field value $\left|\Delta_{0}\right|$. Here we note that at $U=2 t$, the distribution is bimodal at the lowest temperature, showing that there are two different contributions to the superconducting order and the mean amplitude of $\left|\Delta_{i}\right|$ is different at the rim and at the bond sites. This behavior is specific to the bipartite nature of the Lieb lattice. At weak interactions, the effect of the underlying lattice is dominant and thus the distinction of $\left|\Delta_{i}\right|$ at the bond and rim sites show up in the distribution. Distinction between the local order parameters corresponding to the rim and bond sites have been reported in the literature. Based on DMFT calculations it was demonstrated that while the local order parameter corresponding to the rim and bond sites vanish at the same temperature $T_{c}$, their magnitudes are significantly different at $T \neq T_{c}$ [38]. The calculations were however restricted to the weak-coupling regime of upto $U \sim 2 t$. In Fig. 5, we demonstrate how interaction progressively 

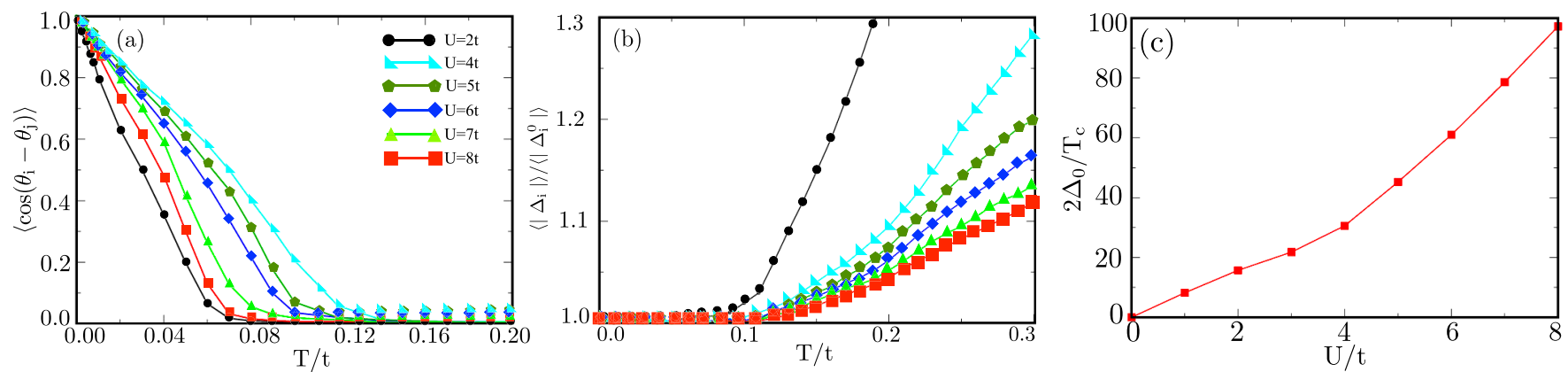

FIG. 4. (a) Thermal evolution of average phase correlation at different interactions $U / t$. The point of inflection in each curve correspond to the $T_{c}$. (b) Thermal evolution of the average pairing field amplitude at different interactions [chosen to be the same as in (a)], normalized by the corresponding value at $T=0$, in each case. (c) Mean-field ratio of superconducting pairing field amplitude $\left(\left|\Delta_{i}^{0}\right|\right)$ at $T=0$ and $T_{c}$. Note the rapid increase in this ratio with increasing $U / t$, which indicates that beyond the weak-coupling regime the mean-field theory severely over estimates the stability of the superconducting state.

renormalizes the distribution, such that for intermediate and strong interactions the distribution is unimodal, indicating uniform contribution to the superconducting order by the bond and rim sites. Progressive rise in temperature broadens out the distribution and shifts the mean amplitude towards larger values of $\left|\Delta_{i}\right|$. Moreover, with increasing interaction the width of the distribution reduces, indicating the reduction in the coherence length of the Cooper pair. In the next section, we demonstrate how this transition in distribution from bimodal to unimodal, bears out in the real space.

\section{Real-space maps}

Figures 6 and 7 show the spatial snapshots of the pairing field amplitude and phase correlation for a single Monte Carlo configuration, as the system evolves in temperature, at different interactions. Each point on the spatial snapshot corresponds to the pairing field amplitude/phase correlation at that site on the Lieb lattice, with the weight indicated by the color coding. In agreement with the bimodal distribution of $\left|\Delta_{i}\right|$ discussed above, at $U=2 t$ and at the lowest temperature the map corresponding to pairing field amplitude shows different magnitude at the rim and bond sites. We note that the pairing field amplitude is large at the bond sites as compared to that of the rim sites. The contribution to the flat band is through the bond sites only, while the rim sites give rise to the dispersive bands. Our real-space maps show that the flat band leads to a larger contribution to superconducting pairing field amplitude as compared to the contribution by the rim sites. The observation is in agreement with the inference drawn on the basis of superfluid weight in Ref. [38], wherein the flat band has been shown to give a larger contribution to the superfluid weight (as geometric weight) as compared to the contribution by the dispersive bands, in the regime of weak coupling. Away from the weak coupling, we find that the contribution to the superconducting pairing field from the flat and dispersive bands are equal. In agreement with the unimodal distribution of $\left|\Delta_{i}\right|$, the real-space map is homogeneous at the lowest temperature, for intermediate and strong coupling. While thermal fluctuations tend to randomize the high-temperature state at all interactions the effect is less pronounced at $U=6 t$, owing to the large superconducting gap.

The long-range phase coherence is robust at the lowest temperature at any finite interaction. Increase in temperature leads to regions where the phase coherence is strongly suppressed. Consequently, there are regions of "local" superconducting phase that survive upto high temperatures leading to the pseudogap phase shown in the phase diagram. Here the system behaves as a collection of Josephson junctions without any phase coherence between them. In the strong-coupling regime, the system loses phase coherence completely at $T>T_{c}$ even though $\left|\Delta_{i}\right|$ continues to be large.

\section{Quasiparticle signatures}

The contribution of the flat and dispersive bands towards superconducting pairing is next analyzed based on the

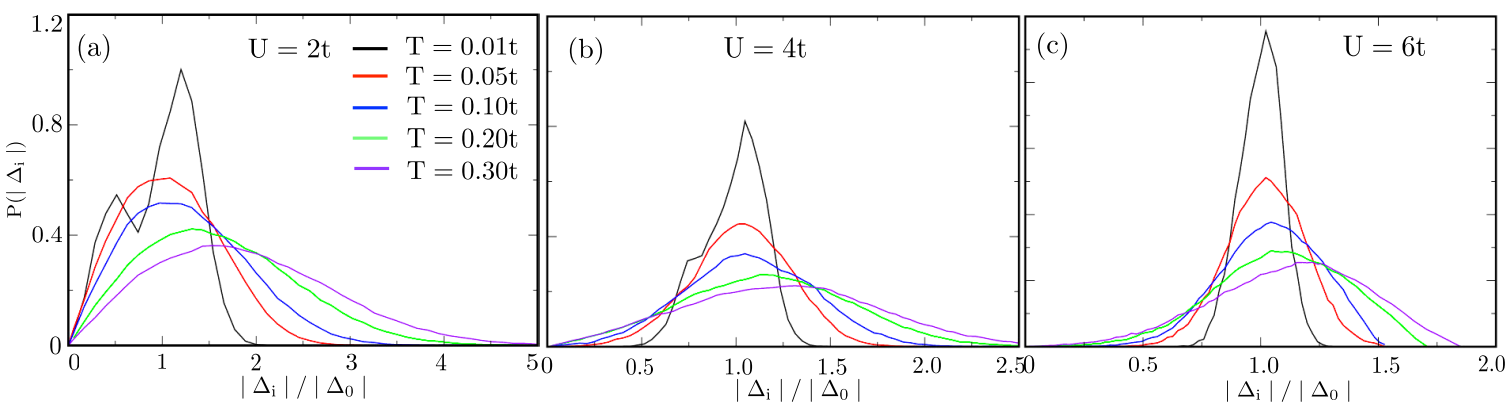

FIG. 5. Distribution of pairing field amplitude $\left|\Delta_{i}\right|$ at different $U / t$, as they evolve in temperature. With increasing temperature the distribution broadens and the mean amplitude of $\left|\Delta_{i}\right|$ shifts towards larger values. 

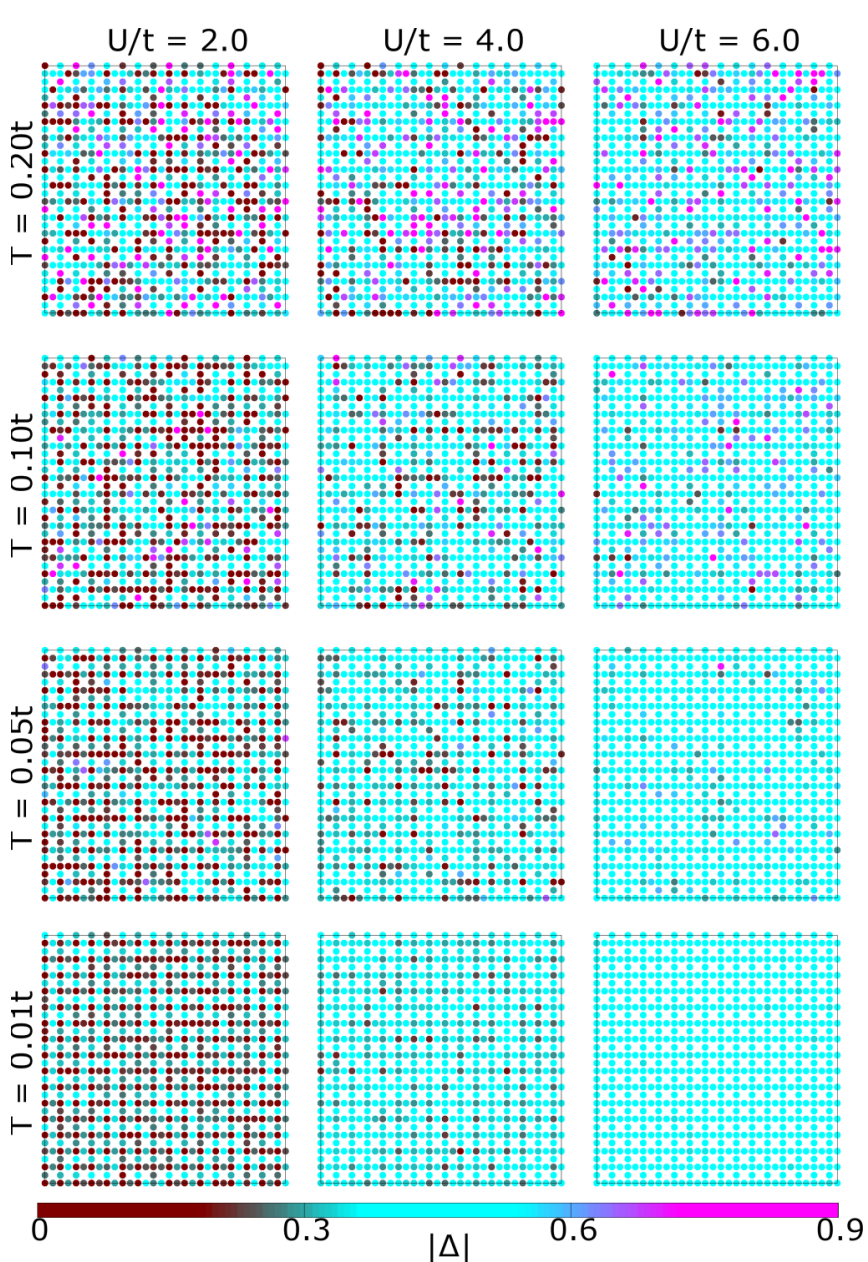

FIG. 6. Real-space maps showing the thermal evolution of the pairing field amplitude $\left(\left|\Delta_{i}\right|\right)$ at different interaction strengths. Note that at weak interactions and at the lowest temperature, the rim and bond sites of the lattice have different magnitudes of $\left|\Delta_{i}\right|$, in agreement with the bimodal distribution of the pairing field amplitude. Each point on the spatial snapshot indicates the pairing field amplitude at that site, with the corresponding weight (magnitude) given by the color bar.

quasiparticle signatures. In Fig. 8, we show the single-particle density of states (DOS) at the selected interaction strengths of $U=2 t, 4 t$, and $6 t$. The low temperature state at any interaction bears signature of $s$-wave superconducting order in terms of a hard gap at the Fermi level and sharp coherence peaks at the gap edges. The prominent satellite peaks away from the Fermi level correspond to the contributions of the spectral weight from the dispersive bands. In the weakcoupling regime of $U=2 t$, thermal fluctuations rapidly pile up spectral weight at the Fermi level and for $T \geqslant 0.1 t$ global superconductivity is lost as indicated by the smearing out of the coherence peaks. The finite spectral weight at the Fermi level signifies the survival of short-range pair correlation, corresponding to the pseudogap phase. The characteristics of spectral weight contributions from the flat and the dispersive bands are very different. The spectral weight contribution of the flat band is independent of momentum, while the dispersive bands exhibits a momentum dependent gap minima.
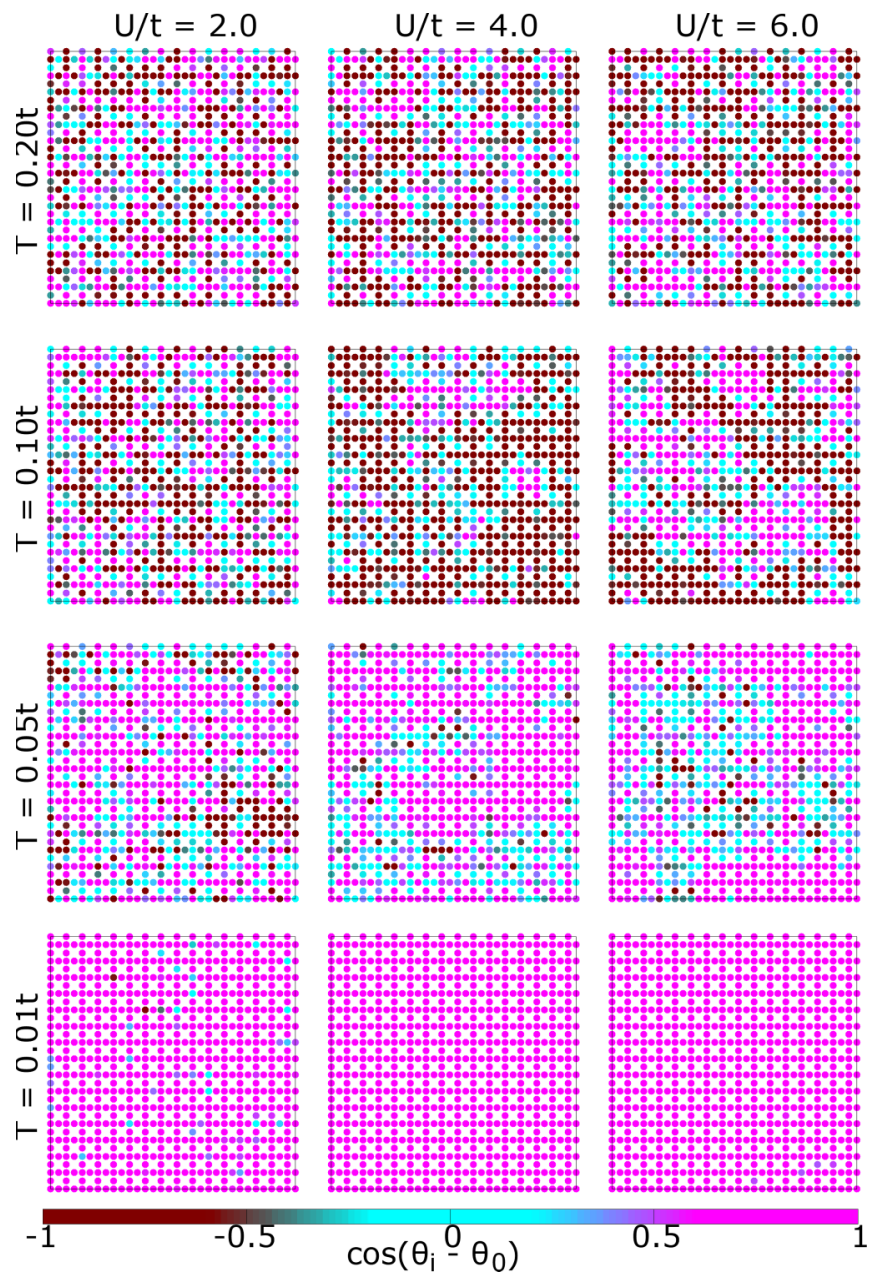

FIG. 7. Real-space maps showing the thermal evolution of the pairing field phase correlation at different interaction strengths. While the low-temperature state corresponds to uniform long-range phase coherence at all interactions, the high-temperature state is spatially phase uncorrelated. Each point on the spatial snapshot indicates the pairing field phase coherence at that lattice site, with the corresponding weight (magnitude) given by the color bar.

In the intermediate coupling regime $(U=4 t)$, interaction renormalizes the band structure and leads to smearing out of the dispersive bands. Consequently, the satellite peaks away from the Fermi level are now less prominent. Upto a temperature of $T \sim 0.05 t$ the superconducting gap at the Fermi level reduces monotonically. At still high temperatures the coherence peaks smear out and there is a small but finite weight at the Fermi level, over the temperature regime of $0.12 t \leqslant T<0.2 t$. The observation is characteristic to the survival of short-range pair correlations in the pseudogap phase. The high-temperature phase manifests strong effect of the flat band localization in terms of immunity of the single-particle DOS at the Fermi level towards thermal fluctuations.

In the regime of strong coupling $(U=6 t)$, the gap persists at the Fermi level even at high temperatures $T \sim 0.3 t$. However, unlike the superconducting gap at the low temperatures, the high-temperature gap is nonsuperconducting and arises out of a strongly correlated bosonic insulating state, as suggested by the absence of coherence peaks. We 

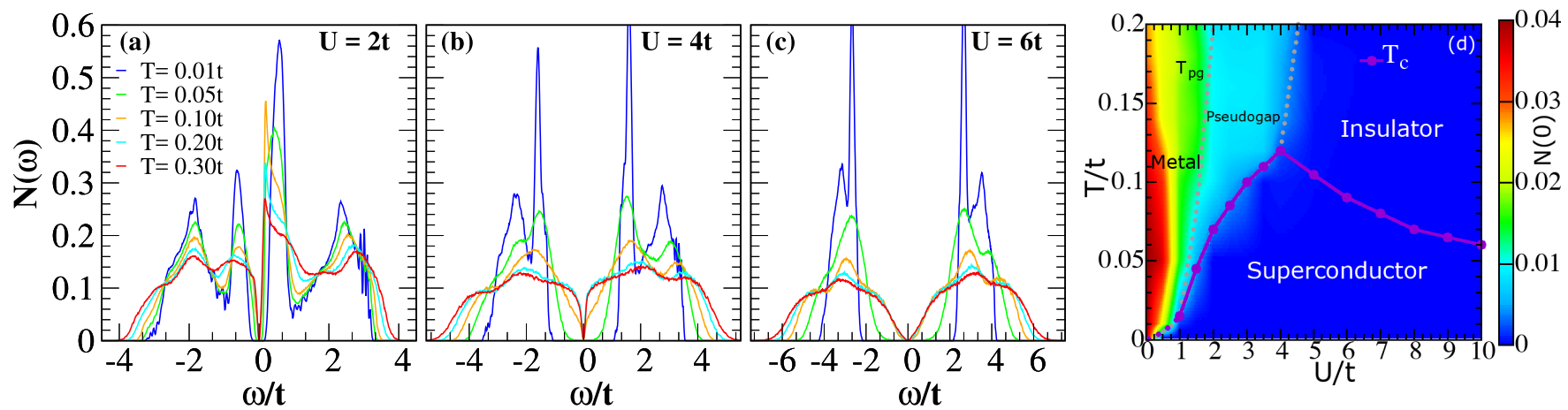

FIG. 8. [(a)-(c)] Thermal evolution of the single-particle density of states (DOS) at different interaction strengths. The loss of superconductivity is indicated by the smearing out of the coherence peaks at high temperatures. Thermal fluctuations pile up spectral weight at the Fermi level giving rise to pseudogap phase at weak and intermediate interactions. The pseudogap regime persists for temperature upto $T \sim 1.5 T_{c}$, indicating the survival of short-range pair correlations even after the long range order is lost. At strong interactions, the DOS is gapped even at high temperatures, though superconductivity is already lost as suggested by the loss of coherence peaks and large transfer of spectral weights away from the Fermi level. The gapped phase at the high temperatures is a correlated bosonic insulator. (d) Map corresponding to the single-particle DOS at the Fermi level, $N(0)$, in the $U-T$ plane. The color indicates the magnitude of the spectral weight.

understand the origin of this high-temperature gap as follows: the strong-coupling regime is characterized by a large pairing field amplitude $\left|\Delta_{i}\right| \sim U$. At low temperatures, this large $\left|\Delta_{i}\right|$ gives rise to a large superconducting gap, as the temperature increases even though the phase coherence is lost the $\left|\Delta_{i}\right|$ continues to be large. Over a narrow window of temperature the $\left|\Delta_{i}\right|$ s changes from being perfectly phase correlated to randomly oriented. These randomly oriented but "large" $\left|\Delta_{i}\right| \mathrm{s}$ not only opens up a gap at the Fermi level, but also broadens the spectra by transferring large weight away from the Fermi level. The contribution to the spectral weight from the dispersive bands have reduced significantly at this interaction as suggested by the vanishing satellite peaks. In Fig. 8(d), we highlight the quasiparticle behavior at the Fermi level in the $U-T$ plane, by mapping out the single-particle DOS $(N(0))$. The $T<T_{c}$ regime correspond to gapped superconducting state as suggested by the vanishing DOS at the Fermi level. In the regime of strong interactions, the high-temperature phase is a correlated bosonic insulator, and thus correspond to a gapped quasiparticle spectrum. The weak interaction regime is characterized by large spectral weight at the Fermi level, corresponding to the metallic phase at high temperature. The pseudogap phase in the intermediate interaction regime is characterized by a small finite spectral weight and a prominent dip, at the Fermi level.

\section{B. Strain-induced superconductor-insulator transition}

In the previous section, we have established the behavior of an $s$-wave superconductor on an unstrained Lieb lattice. Next we focus on the principal aspect of this work, wherein we subject this $s$-wave superconductor to strain, applied through staggered hopping. We have shown that the loss of superconductivity at high temperature is dictated purely by the loss of phase coherence, except at the weak-coupling regime, where the loss of superconductivity is due to the pairing field amplitude fluctuations. We now ask the following questions: what is the fate of this system when the underlying lattice is deformed by applied strain? Whether superconductivity survives in presence of the applied strain or is there a critical strain beyond which the superconducting order is lost? What is the nature of the phases across such a transition? We attempt to answer these questions in the present section.

We select a particular interaction strength of $U=2 t$, such that the effect of the underlying lattice structure is not smeared out by strong interactions, in the BCS-BEC crossover picture and tune the magnitude of applied strain through $\eta$ (see Fig. 2). In the absence of strain $(\eta=0)$ the system at this interaction evolves from a gapped superconducting ground state to a pseudogapped high-temperature phase, with increasing temperature. Straining leads to reconstruction of the band structure of the lattice. We begin our analysis by characterizing this reconstruction of the band structure in terms of (i) spectral line shapes and (ii) spectral function, at the ground state.

\section{Band structure reconstruction}

Figure 9 shows the strain dependent evolution of the momentum resolved spectral function $A(\mathbf{k}, \omega)$ at $U=2 t$. The top panels of Fig. 9 shows the spectral line shape along the trajectory $(0,0) \rightarrow(\pi, 0) \rightarrow(\pi, \pi) \rightarrow(0,0)$ across the Brillouin zone, at selected strain values. The flat band ensures a momentum independent gap at the Fermi level across the momentum trajectory mentioned above. Away from the Fermi level the spectra shows two dispersive bands, which are significantly less robust as compared to the flat band. Increasing strain progressively weakens the coupling between the unit cells, and as a consequence the spectral weight contribution from the dispersive bands reduce. At the same time, the intracell coupling increases with strain, leading the flat band at the Fermi level to renormalize the dispersive bands and flatten them out with increasing strain $(\eta=0.5$ and 0.9$)$. The longrange superconducting order gets progressively destroyed as the unit cells decouple from each other. The large strain regime is thus an insulator with three flat bands, each split into two.

In a Lieb lattice, the dispersive bands touch the flat band at the $M$ point $(\pi, \pi)$ in the Brillouin zone. Consequently, the effect of strain is most pronounced along the trajectory 

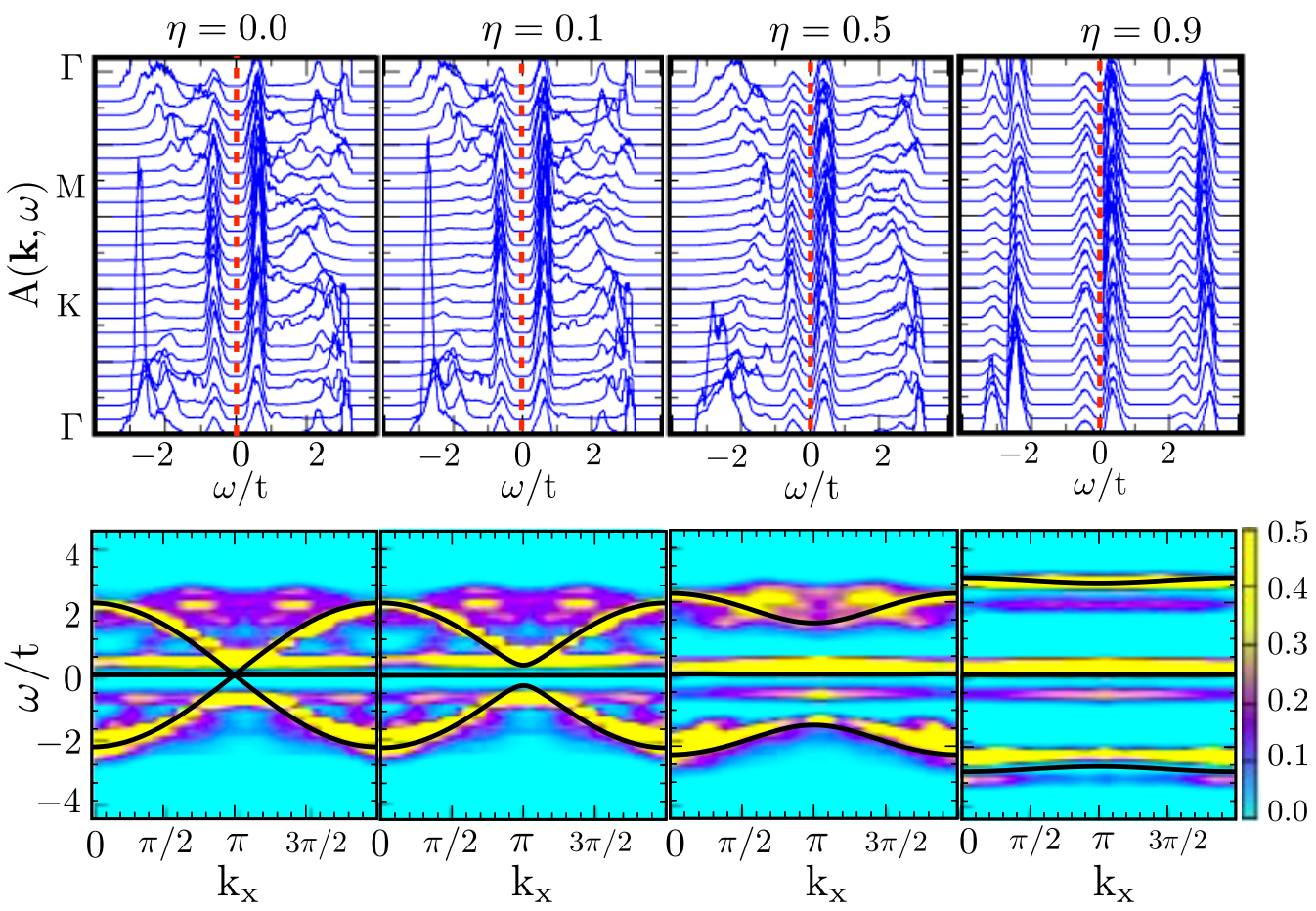

FIG. 9. Quasiparticle signatures at $T=0$ and $U=2 t$ as a function of increasing strain ( $\eta$ ), expressed in terms of the (i) spectral line shape and (ii) spectral function $[A(\mathbf{k}, \omega)]$. The top row shows the spectral line shape along the trajectory $(0,0) \rightarrow(\pi, 0) \rightarrow(\pi, \pi) \rightarrow(0,0)$ across the Brillouin zone. Note that the flat band at the Fermi level remains immune to the effect of strain, however the dispersive bands away from the Fermi level progressively flattens out with increasing $\eta$. In the bottom row, the spectral function evolution along the trajectory $\left(q_{x}, \pi\right)$ is shown as the function of increasing strain. At large strain, the spectrum comprises of localized energy bands only. The dispersion spectra as obtained in the noninteracting limit $(|U|=0)$ is shown as solid black curves on the maps. The effect of interaction is to open up a gap at the Fermi level.

containing the $M$ point. We show the spectral function maps along the $\left(k_{x}, \pi\right)$ trajectory as they evolve with strain, in the bottom row of Fig. 9. Further, we compare the dispersion spectra with that obtained at the noninteracting limit (black curves) so as to highlight the effect of interactions on the system. In the noninteracting and unstrained limit $(\eta=0)$, the dispersion spectra is analytically tractable and is given as

$$
E_{ \pm \mathbf{k}}= \pm 2 t \sqrt{1+\left(\cos k_{x} a+\cos k_{y} a\right) / 2} .
$$

Application of strain progressively pushes the dispersive bands away from the flat band and flattens them out. The corresponding dispersion relation reads as

$$
E_{ \pm \mathbf{k}}= \pm 2 t \sqrt{1+\eta^{2}+\left(1-\eta^{2}\right)\left(\cos k_{x} a+\cos k_{y} a\right) / 2}
$$

where $a$ is the lattice spacing [38]. The flat band by itself is immune to strain and unlike graphene does not undergo a gap opening just by the application of strain. Interaction opens up a gap at the Fermi level irrespective of the magnitude of the strain and choice of the trajectory across the Brillouin zone. As the strain is increased and the dispersive bands are pushed away from the flat band, they split into two as they flatten out progressively with strain. In the limit of large strain $(\eta=0.9)$, the spectra comprises of three flat bands (each split into two) with localized energy. The dispersion spectra carries crucial information regarding the momentum dependence of the underlying superconducting state.
As mentioned before there are two separate contributions to the superconducting order, arising out of the flat and the dispersive bands. A momentum dependent spectra with a finite momentum gap minima is a signature of BCS-like superconducting state [56,57]. While on the other hand a spectral gap at $\mathbf{k}=0$ corresponds to a superconducting state akin to BEC. As the name suggests, the spectral weight contribution of the flat band is always independent of momentum. In the superconducting regime $\left(\eta<\eta_{c}\right)$, the contribution of the dispersive bands undergo a crossover akin to the BCS-BEC, as a function of increasing strain, such that the pairing field amplitude remains robust against the applied strain but the phase coherence is progressively lost. Based on the inferences we draw from the strain-induced band structure reconstruction, we suggest that close to the SIT the scenario is dominated by the spectral weight contribution from the flat band. It is tempting to call this contribution as "bosonic," owing to the lack of finite momentum dependence of the spectra. In that spirit, the spectral weight contribution from the flat band is always bosonic, even in the unstrained limit. In the limit of large interaction (at $\eta=0$ ), the dispersive bands merge with the flat band giving rise to a momentum independent dispersion spectra, and in turn a bosonic contribution. The crossover from a BCS like physics to a BEC like state with respect to either $\eta$ or $U$ is always determined by the contribution of the dispersive bands. It must however be kept in mind that the indicators discussed in this work are based on (i) single-particle correlations and (ii) MFT like approach towards quantum 


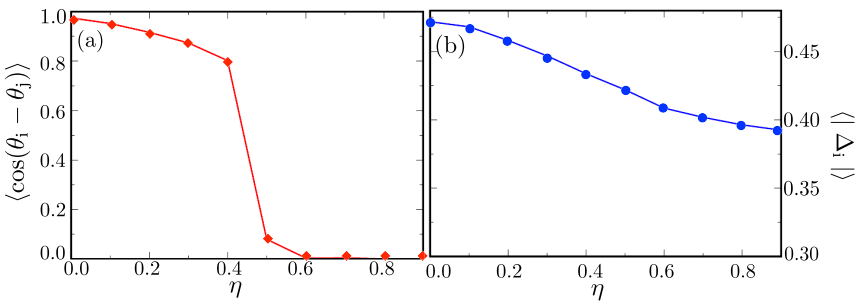

FIG. 10. (a) Average pairing field phase correlation and (b) average of pairing field amplitude at $T=0$ as a function of increasing strain $(\eta)$ at $U=2 t$. The system loses its long-range phase coherence at $\eta=\eta_{c} \sim 0.6$ as shown by (a). The pairing field amplitude however survives at $\eta>\eta_{c}$.

phase transition. Whether a more sophisticated approach to this problem via DQMC, characterized through two particle correlations modify this picture is an intriguing aspect worth investigating, but is out of the scope of the present work.

\section{Global indicators}

We next show the global indicators in terms of average phase correlation and average pairing field amplitude as a function of strain at the ground state, in Fig. 10. We note that while the long-range phase coherence is destroyed by strain, the pairing field amplitude is only weakly suppressed. This is a significant observation, which shows that even at the ground state it is the loss of long-range phase coherence that kills off the superconducting order. Recent DMFT calculations have suggested that the loss of superconductivity at a critical strain is dictated by the collapse of local superconducting order parameter [38]. We argue that the critical strain should be determined based on the loss of long-range phase coherence and that the pairing field amplitude is always finite. It would be interesting to probe the superconducting order locally for intermediate regime of strain (close to $\eta_{c}$ ) where even though the pairing field amplitude is robust, the (quasi) long-range phase coherence is strongly suppressed. This can be achieved via scanning tunneling microscopy (STM) and tunnelling conductance measurements which can probe the spatial order locally. One would expect a progressive disappearance of the coherence peaks in the local density of states, with increasing strain. Figure 10 shows that at $U=2 t$ the system undergoes a quantum superconductor-insulator transition at the critical strain of $\eta_{c} \sim 0.6$.

\section{Thermal behavior}

We now investigate the fate of the strain-induced superconductor-insulator transition at finite temperature. For this we present the thermal evolution of the average phase correlation at different magnitudes of strain, in Fig. 11(a). As before, the point of inflection corresponds to the loss of longrange phase coherence, i.e., $T_{c}$. We note that increasing strain leads to progressive suppression of $T_{c}$. The corresponding distribution of the pairing field amplitude at selected strain are shown in the panels (b)-(d) of Fig. 11. We note that the distribution is bimodal at the lowest temperature for any strain, indicating the difference in the contribution of the bond

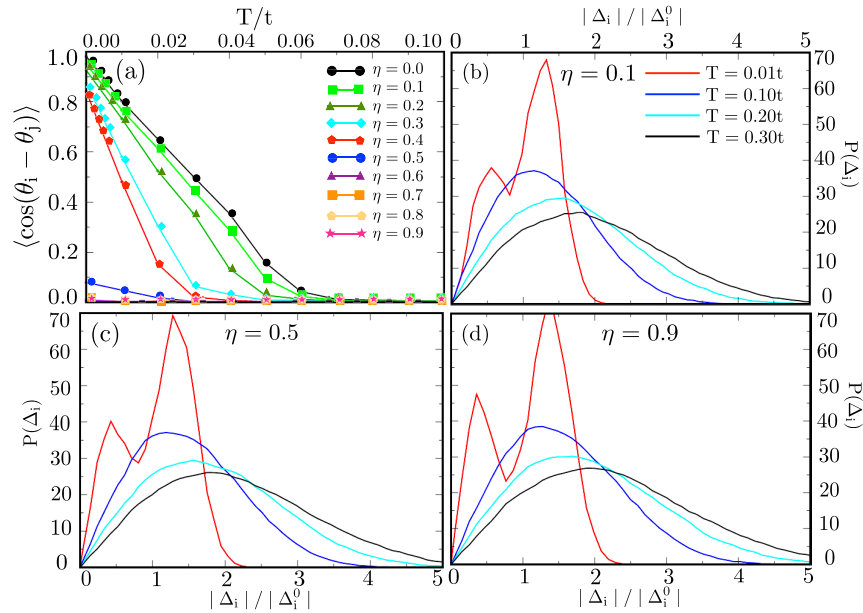

FIG. 11. (a) Thermal evolution of average pairing field phase correlation with increasing strain $(\eta)$ at $U=2 t$. The point of inflection in each curve indicates the $T_{c}$. Note how phase correlation is strongly suppressed beyond $\eta \sim 0.4$ and is lost at $\eta_{c} \sim 0.6$. The distribution of the pairing field amplitude at selected strain cross sections of $\eta=0.1,0.5$, and 0.9 are shown in (b)-(d), respectively. At the lowest temperature the distribution is bimodal at all strain regimes, showing that the bond and rim sites of the underlying lattice contains pairing fields of different amplitudes. Thermal fluctuations smooth out the distributions and makes them unimodal. With increasing temperature the mean amplitude of the pairing field shifts towards larger values, at all strains. Note that even in the insulating regime the pairing field amplitude remains fairly robust.

and rim sites towards the superconducting pairing. While for the unstrained case we had shown that increasing interaction tends to homogenize the contribution from the bond and rim sites, Fig. 11 suggests that increasing strain leads to a larger difference between the contribution from the bond and rim sites. In other words, strain enhances the bipartiteness of the lattice. We note from the distributions that the contribution towards superconducting pairing field amplitude from the flat band is $\sim 1.5$ times of that from the dispersive bands. The simple reason for the same is the localization of large number of energy states by the flat band. As expected, temperature randomizes the pairing field amplitude and leads to a broader distribution. Moreover, the peak amplitude shifts towards larger values in agreement with the fact that thermal fluctuations enhance the mean pairing field amplitude.

\section{Real-space maps}

The real-space maps corresponding to the pairing field amplitude and phase correlation shown in Figs. 12 and 13 bear out the information presented through the distributions. At the lowest temperature the contribution to the pairing field principally arises from the flat bands located at the bond sites, irrespective of the magnitude of strain. Increasing temperature randomizes the pairing field amplitude leading to isolated islands of suppressed or enhanced pairing. We note that even at large strain $(\eta=0.9)$, the magnitude of $\left|\Delta_{i}\right|$ s remain fairly robust over a large fraction of the lattice, ensuring a gapped quasiparticle spectra. 


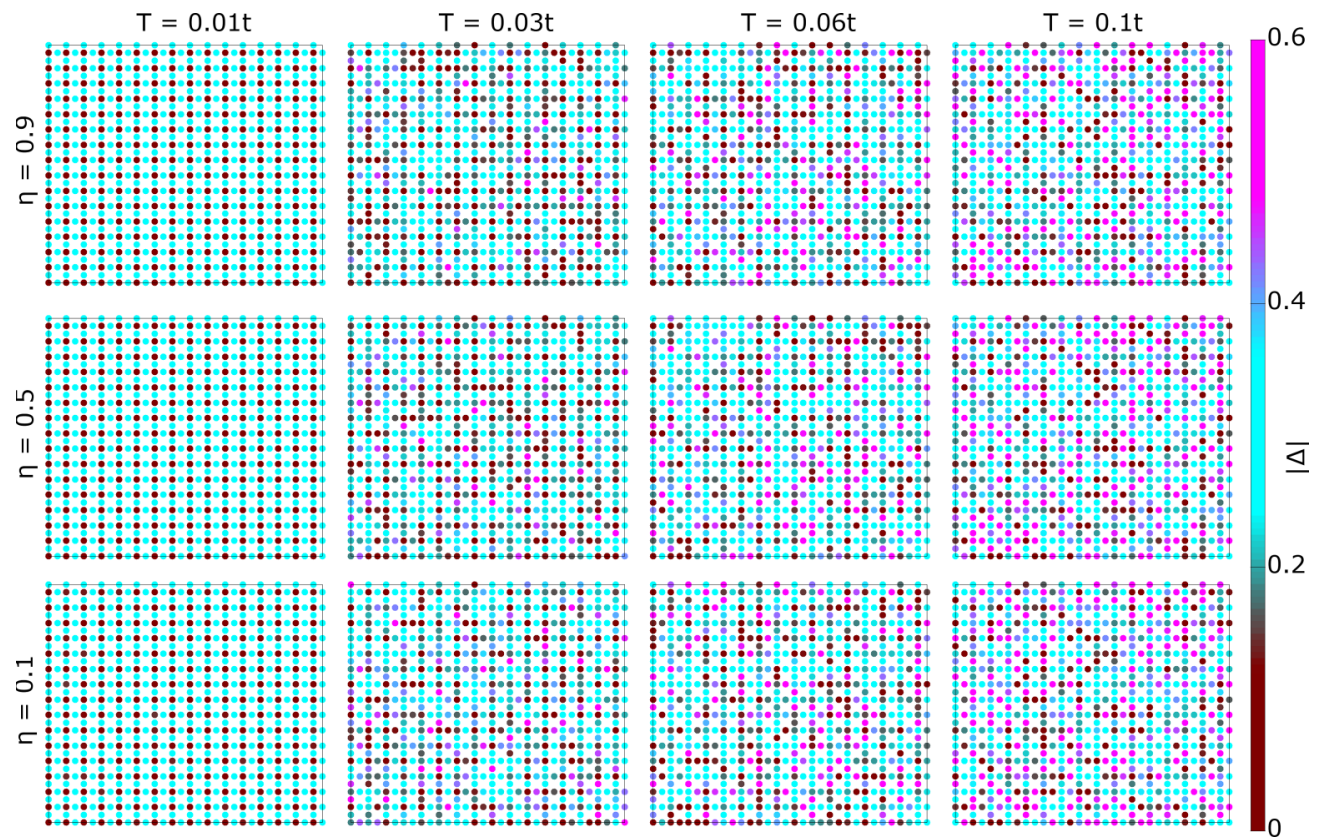

FIG. 12. Real-space snapshots of pairing field amplitude as it evolve with temperature at $U=2 t$ and selected strain cross sections. The lowest temperature phase shows bimodal distribution of $\left|\Delta_{i}\right|$ at all $\eta \mathrm{s}$. Each point in the map indicates the pairing field amplitude at that particular lattice site with the corresponding weight (magnitude) being given by the color bar.

In contrast to the pairing field amplitude, the long-range phase coherence is rapidly suppressed with strain, as demonstrated in Fig. 13. At weak strain $(\eta=0.1)$, the system has long-range phase coherence at the lowest temperature, which survives upto intermediate temperatures $\left(T \sim T_{c}\right)$. For
$T>T_{c}$, isolated regions of suppressed phase coherence are realized in the system. The phase is akin to the pseudogap regime discussed before, where short-range pair correlations survive without long-range order. Further rise in temperature at this strain randomizes the phase completely, leading to
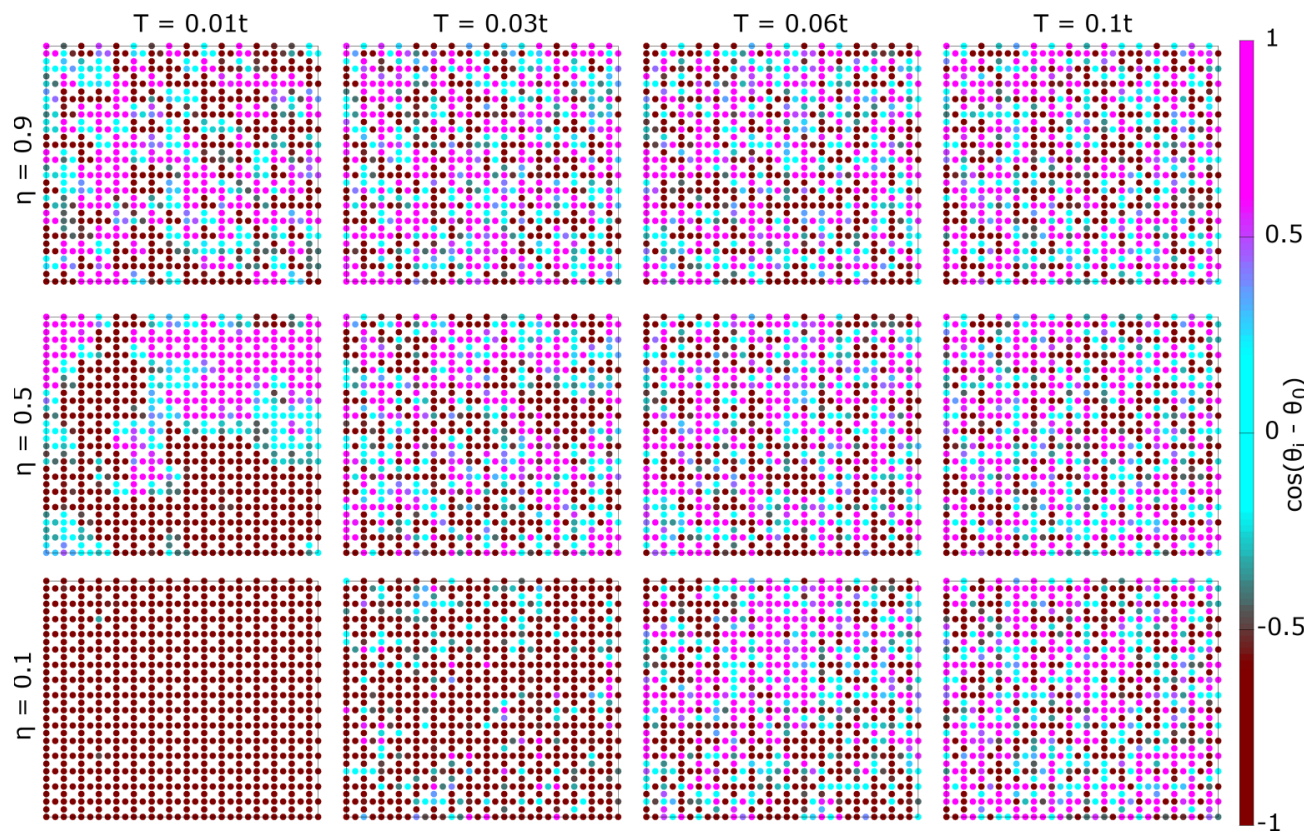

FIG. 13. Real-space snapshots of pairing field phase correlation as it evolve with temperature at $U=2 t$ and selected strain cross sections. At weak strain $(\eta=0.1)$, the phase correlation is long-ranged and uniform, which progressively randomizes with temperature. The intermediate strain regime of $\eta=0.5$ shows spatial suppression of phase coherence at isolated regimes even at the lowest temperature, suggesting weakening of the superconducting order. The large strain regime $(\eta=0.9)$ is an insulator as suggested by the absence of phase coherence even at the lowest temperature. Each point in the map indicates the pairing field phase correlation at that particular lattice site with the corresponding weight (magnitude) being given by the color bar. 

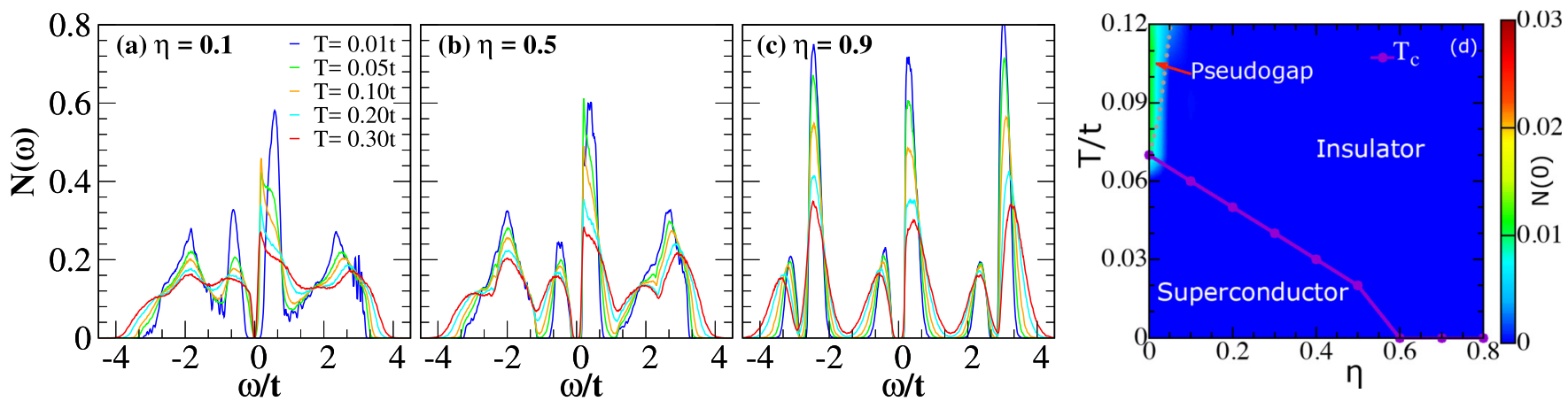

FIG. 14. [(a)-(c)] Thermal evolution of single-particle density of states (DOS) at $U=2 t$ and selected $\eta$ values. At small strain ( $\eta=0.1)$, the system undergoes a transition from a gapped superconductor to a pseudogapped regime with increasing temperature. At intermediate $(\eta=0.5)$ and strong $(\eta=0.9)$ strain, the gap at the Fermi level is immune to thermal evolution, owing to the flat band. Additionally, away from the Fermi level the DOS gaps out, indicating the localization of the states. (d) Map corresponding to the single-particle DOS at the Fermi level, $N(0)$, across the $\eta-T$ plane, at $U=2 t$. The magnitude of the spectral weight is indicated by color.

complete loss of superconductivity. At intermediate strain $(\eta=0.5)$ the phase coherence is significantly suppressed even at the lowest temperature. Consequently, the corresponding $T_{c}$ is suppressed. Increase in temperature leads to rapid loss of phase coherence, thereby killing off superconductivity. Note that at this strain there is no pseudogap regime. The large strain $(\eta=0.9)$ regime lacks any phase coherence even at the lowest temperature. As a result, this regime can be broadly thought of to be a insulating state. Thermal fluctuations do not affect the state significantly. The combined picture that emerges from the pairing field amplitude and phase coherence at large strain is that of a bosonic insulator. Such an insulator is characterized by (i) finite pairing field amplitude, (ii) lack of phase coherence, and (iii) large single-particle spectral gap at the Fermi level. While (i) and (ii) are shown in Figs. 12 and 13, we discuss (iii) in the next section pertaining to quasiparticle behavior.

\section{Quasiparticle signatures}

In Fig. 14, we show the thermal evolution of single-particle density of states (DOS) at selected temperature and strain values, at $U=2 t$. Figure 14(a) shows the behavior at weak strain of $\eta=0.1$. The low-temperature phase corresponds to a gapped BCS-like superconductor as suggested by the sharp coherence peaks at the gap edges. There are prominent satellite peaks corresponding to the two dispersive bands away from the Fermi level. Increasing temperature leads to progressive piling up of spectral weight at the Fermi level along with the smearing out of the coherence peaks, leading to a weak pseudogap like behavior. The loss of superconductivity in this case is signalled by the smearing out of the coherence peaks for $T \geqslant 0.06 t$. In Fig. 14(b), we show the thermal evolution of the DOS at an intermediate strain $(\eta=0.5)$. The lowest temperature state at this parameter regime is a (BEC-like) superconductor with a finite gap at the Fermi level. Note that the gap at the Fermi level is nearly immune to thermal fluctuations. The satellite peaks away from the Fermi level gets progressively robust with increasing strain, as the energy states get localized. At large strain, $\eta=0.9$, the unit cells get nearly decoupled from each other [see Fig. 14(c)]. The dispersive bands flatten out and the spectra now comprises of three flat bands (each split into two) with localized energy states. Long-range superconducting order is lost in this regime and the system behaves as a bosonic insulator.

In Fig. 14(d), we show the single-particle DOS at the Fermi level, $N(0)$, at $U=2 t$, in the $\eta-T$ plane. While the flat band leads to a finite gap across most of the $\eta-T$ plane, in the regime of weak strain, a small window of pseudogap phase emerges at high temperatures, as signalled by the nonzero spectral weight at the Fermi level. The strain-induced SIT discussed in this paper is generic for $x-y$ anisotropy in the Lieb lattice. The $N(0)$ map shows that over a large part of the parameter space the system undergoes transition from a bosonic superconductor to a bosonic insulator.

\section{DISCUSSION AND CONCLUSION}

In this paper, we have shown a superconductor-insulator quantum phase transition, driven by strain. Across a critical strain $\eta_{c}$, we have characterized this SIT based on thermodynamic signatures. We have compared and contrasted our inferences vis a vis with those drawn based on the behavior of superfluid weight [38]. Our results suggest that the loss of superconducting order with increasing strain is dictated by the loss of long-range phase coherence even though local pair correlations survive. We have further demonstrated a strain-induced BCS-BEC like crossover in the regime of weak interaction, in this system. Moreover, in agreement with the inference drawn based on the behavior of superfluid weight, our results show that the bond sites of the lattice gives larger contribution to superconducting pairing as compared to that of the rim sites. Applying strain is a novel but certainly not an unique route to realize SIT. A more conventional route is disorder driven SIT. While the final outcome is similar through both the approaches, the underlying mechanisms are very different. We touch upon them below.

\section{A. Disorder versus strain-induced SIT}

Disorder (as introduced via the randomness of chemical potential in the system) induced SIT is a well studied subject [58-62]. The fundamental observation in case of disorderinduced SIT is the fragmentation of the superconducting state 
(both in superconducting amplitude and phase correlation) as a function of increasing disorder. The resulting "insulating" state is basically a nontrivial state with localized pairs. The single-particle spectra at the Fermi level remains gapped, in spite of the Griffith's effects of the rare regions [58-60]. The two particle gap $\omega_{\text {pair }}$, on the other hand is not a hard gap $[60,63]$.

On the other hand for a strain-induced (disorder free) SIT, there is no spatial inhomogeneity in the superconducting amplitude across the transition. Both the superconductor and insulator phases are characterized by large and homogeneous pairing field amplitude corresponding to a BEC-like state and a bosonic insulator, respectively. The system discussed in this manuscript falls in this category as is made evident through the spatial maps of pairing field amplitude and phase correlation. The single-particle spectra is hard gapped across the phase transition. Discussion of two particle gap is beyond the scope of this work, however, existing literature on disorder free transition suggests that the two-particle gap $\omega_{\text {pair }}$ is hard gapped across such SIT [56]. Investigation of the effect of flat bands on the two-particle gap is a subject worth pursuing in future.

\section{B. Connection with experiments}

In the context of solid state materials, the survival of local pair correlations can be probed through STM measurements and tunneling conductance maps. Recently, it has been found that two-dimensional (2D) organic system of $s p^{2}$ carbon-conjugated covalent organic framework $\left(s p^{2} c\right.$-COF) is a material realization of the Lieb lattice [64]. Based on the experimental observation [64] and numerical simulations [65], it was found that the material has a Lieb lattice like structure with staggered hopping (strain), similar to the situation discussed in the present work. $s p^{2} c$-COF has been experimentally observed to exhibit metal insulator transition as well as unconventional magnetic instability, which were attributed to the strained lattice structure of this material [64]. Such a discovery of material realization of Lieb-like lattice indeed opens up scope for future exploration for systems exhibiting novel phase transitions and unconventional quantum phases. One such "yet to be discovered" possibility is the SIT discussed in the present paper.

While the application of strain through staggered hopping is an experimentally challenging task for solid state materials, it is certainly more feasible for ultracold atomic gases and photonic lattices, where the control parameters are tunable. As on today, one of the biggest challenges faced by the ultracold atomic gas experiments is that sufficiently low temperatures could not be attained so as to realize superfluid ground state. However, capturing the signatures of pair correlations at high temperatures is certainly an achievable goal for these experiments and has already been done for Fermi gases [66,67], population imbalanced ultracold atomic gases $[68,69]$, etc. The spectroscopic probes to capture the signature of preformed pairs in these systems are radio frequency (rf) spectroscopy, momentum resolved $\mathrm{rf}$ spectroscopy, etc. [66-82]. We believe that similar techniques can be utilized to understand the physics of superfluid pair formation in ultracold atomic gases on Lieb lattice. The finite temperature results pertaining to the quasiparticle signatures discussed in this paper are expected to provide useful benchmarks for the experiments based on spectroscopic measurements.

Finally, ours is not the first system to show disorder free BEC-bosonic insulator SIT at weak coupling [56]. However, to the best of our knowledge, we have demonstrated the same for the first time for a flat band system. Moreover, not only this system has been realized as an artificial designer lattice, recently a solid state material with similar lattice architecture has been realized. This makes our predictions easily verifiable in experimental settings.

The SIT discussed in this manuscript is not a consequence of the flat band in the system. It is the band structure reconstruction (change in topology of the bands) due to the applied strain which gives rise to the SIT. The spectral and real-space signatures show that there are bosonic phases on the either side of the SIT (BEC and bosonic insulator), even in the weak coupling regime. Our results suggest that the nature of the superconducting pairing field as contributed by the flat and dispersive bands might be different. While the contribution of spectral weight by the flat band is always bosonic, the dispersive band contributions depend on the applied strain and/or interactions, which facilitates the BCS-BEC crossover like physics. Our analysis further shows that the exclusion of phase fluctuations of the pairing field in the calculation of this SIT would lead to an incorrect phase diagram. Since the pairing field amplitude remains robust across the transition, mapping out the phase diagram based on it would show the survival of superconducting order even for an arbitrarily large strain.

In conclusion, in this work, we have established the straininduced superconductor-insulator quantum phase transition on the Lieb lattice. Based on thermodynamic signatures, we have demonstrated how global superconductivity is destroyed with loss of long-range phase coherence and also presented a mean-field estimate of critical strain across which the quantum phase transition takes place between a bosonic superconductor and a bosonic insulator. Further, we have demonstrated that strain alters the characteristic of the underlying superconducting order and leads to a strain-induced BCS-BEC crossover. This work is the first demonstration of disorder free SIT on the Lieb lattice and is expected to open up experimental avenues both for the solid state and ultracold atomic gas communities. Further, we have discussed several indicators which we believe should be accessible through the existing experimental probes. Apart from being engineered in the artificial systems such as photonic lattices or ultracold atomic gases, the Lieb lattice has been found to be the building blocks of solid state materials such as cuprates, 2D organic materials etc. Such materials require a more complex model than the one discussed in this paper. While it is nontrivial to include all the interactions and parameters relevant for such materials in a tractable theoretical model, the future works aim towards capturing the physics of these materials through more realistic models.

\section{ACKNOWLEDGMENTS}

N. S. acknowledges use of the National Supercomputing Centre (NSCC) Singapore ASPIRE-1 cluster for some part 


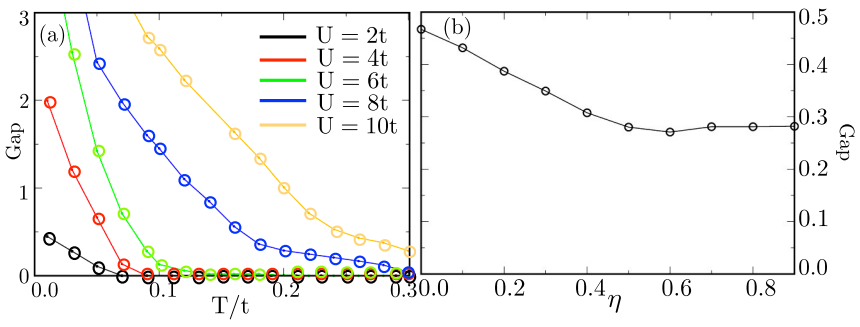

FIG. 15. (a) Thermal evolution of quasiparticle spectral gap at selected interactions, for an unstrained Lieb lattice. (b) Evolution of spectral gap as a function of strain across the quantum phase transition at $\eta_{c}=0.6$ and $U=2 t$.

of the numerical simulations. M. K. acknowledges use of the HPC clusters at Harish Chandra Research Institute, India for detailed numerical calculations.

\section{APPENDIX A: SUPERCONDUCTING GAP}

In the earlier sections, we have discussed that the loss of superconductivity is dictated by the loss of long-range phase coherence, even though the pairing field amplitude remains finite. While a mean-field treatment is expected to overestimate the robustness of the ordered state, a recent comparison between the mean-field and DMFT results suggest that in the limit of weak coupling and low temperatures the agreement between the two approaches is reasonable [38]. At higher temperatures, the DMFT treatment expectedly fares better since it takes into account some of the spatial fluctuations. Stronger interactions tend to make the approach less accurate (for $d<\infty$ ) as spatial fluctuations dominate the high-temperature scenario. The characterization of the superconducting state in Ref. [38] is carried out based on the local (sublattice) superconducting "order parameter," determined by solving the self consistent gap equation. In our present numerical scheme, the global superconducting gap can be determined from the single-particle DOS at the Fermi level and we show the same in Fig. 15. Panel (a) of Fig. 15 corresponds to the thermal evolution of the superconducting gap at different interaction strengths corresponding to the weak, intermediate and strong-coupling regimes. We note that in the regime of weak interactions the behavior of the gap is in agreement with the one obtained from DMFT calculations with the gap closing at the $T_{c}$ [38]. At stronger interactions however the gap is robust even at higher temperatures. We emphasize that the high-temperature gap correspond to a correlated bosonic insulator rather than a superconductor. In other words, away from the weak-coupling regime, the superconducting order is no longer tied to the quasiparticle spectral gap at the Fermi level indicating the breakdown of the mean field theory. Though the results corresponding to DMFT analysis in Ref. [38] pertains to the weak interaction regime only, we believe that neglect of spatial fluctuations would lead to incorrect thermal scales away from the weak coupling within the framework of DMFT.

Figure 15(b) shows the evolution of quasiparticle spectral gap at the Fermi level with strain at the ground state and $U=2 t$. For $\eta \leqslant \eta_{c}$, there is monotonic decrease in the gap with increasing strain since the superconducting correlations

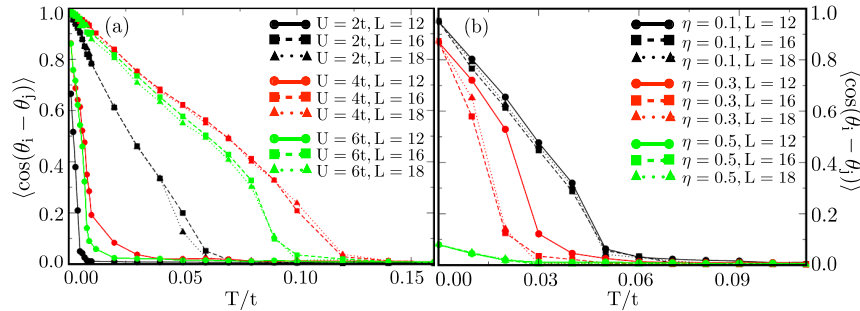

FIG. 16. (a) Thermal evolution of average phase correlation at selected interactions of $U=2 t, 4 t, 6 t$ and system sizes of $L=12$, 16, and 18. (b) Thermal evolution of average phase correlation at $U=2 t$ and selected strain values of $\eta=0.1,0.3$, and 0.5 for the system sizes same as in (a).

weaken as the unit cells progressively decouple from each other with increasing strain. For strain $\eta>\eta_{c}$, the system is in an insulating state and the corresponding spectral gap at the Fermi level is immune to the effect of strain. The change in the size of the gap across the SIT is $\sim 18 \%$. Note that the spectral gap does not vanish with increasing strain, rather it is the phase correlation which undergoes transition across the quantum critical point.

\section{APPENDIX B: FINITE SYSTEM SIZE EFFECT}

The results discussed in this paper correspond to a system size of $N=768(L=16)$. Any lattice simulation is, however, susceptible to finite size effects and in order to verify whether our results are robust against the choice of the system size we have carried out the numerical simulations at different system sizes. In Fig. 16, we show the thermal evolution of superconducting phase correlation at, (a) selected interactions $U=2 t, 4 t$, and $6 t$ (for the unstrained case) and (b) selected strain values $\eta=0.1,0.3$, and 0.5 (for the strained case) at three different lattice sizes of $N=432(L=12), N=$ $768(L=16)$, and $N=972(L=18)$. We note that while there are noticeable finite size effect at $N=432$ as observed via the underestimation of the $T_{c}$ scales, the larger system sizes $N \geqslant 768$ are immune to finite size effects, suggesting that the results discussed in this paper are fairly robust against the choice of the system sizes. Our finite system size analysis further justifies that the strongly suppressed $T_{c}$ at strong coupling $(U=8 t)$ as observed in DQMC study [55] is an artifact of small system size.

\section{APPENDIX C: FIXED CHEMICAL POTENTIAL VERSUS FIXED NUMBER DENSITY}

Our calculations are carried out in grand canonical ensemble with a fixed chemical potential of $\mu=-0.2 t$. In many situations such as inclusion of disorder in the system, the fermionic number density varies significantly with the disorder strength/concentration in calculations carried out in grand canonical ensemble. The inferences that one would draw from such a calculation will be significantly different from those obtained from the calculations carried out at fixed number density. In order to understand the dependence of the number density on temperature, interaction and strain at $\mu=$ 

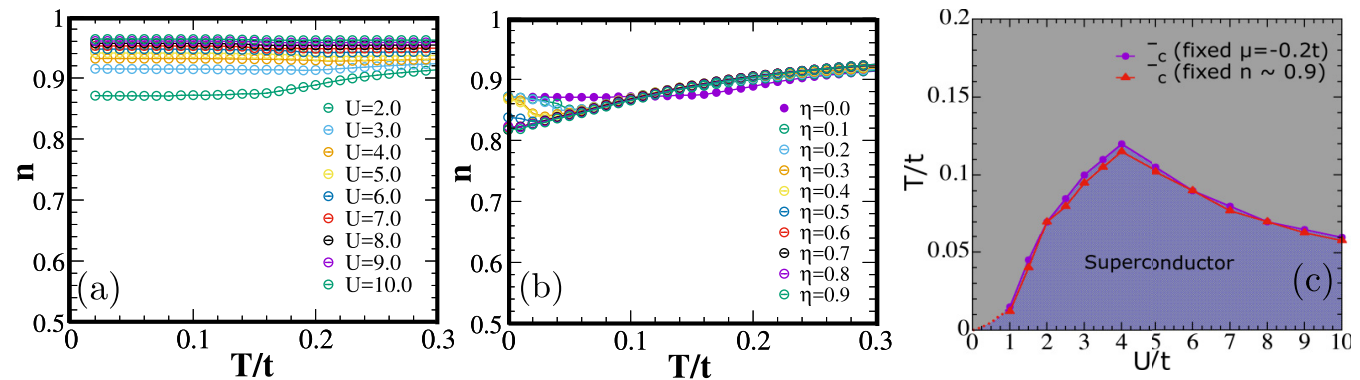

FIG. 17. Thermal evolution of fermionic number densities at different (a) interactions and (b) strain. (c) BCS-BEC crossover at $\eta=0.0$ as computed at a fixed chemical potential $(\mu=-0.2 t)$ and a fixed number density $(n \approx 0.9)$.

$-0.2 t$, we show the same in Fig. 17. The choice of $\mu=-0.2 t$ corresponds to a fermionic filling of $n \approx 0.9$ and for $|U| \geqslant 2 t$ remains almost independent of the choice of the interaction strength and temperature. Importantly, in the absence of any competing order at the low temperature (as in the present model), we do not expect a small drift in the fermionic number density to alter the state of the system significantly. We have verified the same across the BCS-BEC crossover and the results are shown in Fig. 17(c). The figure shows the comparison of the BCS-BEC crossover as calculated at a fixed chemical potential of $\mu=-0.2 t$ and at a fixed fermionic number density of $n \approx 0.9$. The results suggest that for the system (model) and parameter regime under consideration, calculations carried out in grand canonical ensemble captures the behavior of the system accurately.

\section{APPENDIX D: STATIC PATH APPROXIMATION (SPA) IN COMPARISON WITH OTHER TECHNIQUES}

The Hubbard model at intermediate interaction requires a nonperturbative solution. The exponential growth in the dimension of the Hilbert space rules out the use of exact diagonalization except for very small system sizes. The "exact" tool of choice is quantum Monte Carlo (QMC) and all approximations are bench marked against it. While QMC can be implemented via various approaches, the method below easiest reveals the connection to our approach $[47,48,50]$.

The Hubbard partition function is written as a functional integral over Grassmann fields $\psi_{i \sigma}(\tau), \bar{\psi}_{i \sigma}(\tau)$ as

$$
\begin{aligned}
Z= & \int \mathcal{D} \psi \mathcal{D} \bar{\psi} e^{-S[\psi, \bar{\psi}]}, \\
S= & \int_{0}^{\beta} d \tau\left[\sum_{i j, \sigma, \sigma^{\prime}}\left\{\bar{\psi}_{i \sigma}\left(\left(\partial_{\tau}-\mu\right) \delta_{i j}-t_{i j}\right) \psi_{j \sigma}\right\}\right. \\
& \left.+|U| \sum_{\langle i j\rangle, \sigma, \sigma^{\prime}} \bar{\psi}_{i \sigma} \psi_{i \sigma} \bar{\psi}_{j \sigma^{\prime}} \psi_{j \sigma^{\prime}}\right] .
\end{aligned}
$$

Only quadratic path integrals can be exactly evaluated. Since the interaction generates a quartic term in the $\psi$, the partition function cannot be immediately evaluated.

The quartic term is "decoupled" exactly through a Hubbard-Stratonovich transformation in terms of pairing fields $\Delta_{i}(\tau), \bar{\Delta}_{i}(\tau)$. This induces a term $\Delta_{i} \bar{\psi}_{i \uparrow}(\tau) \bar{\psi}_{i \downarrow}(\tau)$ in the action

$$
\begin{aligned}
Z= & \int \mathcal{D} \Delta \mathcal{D} \Delta^{*} \mathcal{D} \psi \mathcal{D} \bar{\psi} e^{-S_{1}\left[\psi, \bar{\psi}, \Delta, \Delta^{*}\right]}, \\
S_{1}= & \int_{0}^{\beta} d \tau\left\{\sum_{i j, \sigma}\left[\bar{\psi}_{i \sigma}\left(\left(\partial_{\tau}-\mu\right) \delta_{i j}-t_{i j}\right) \psi_{j \sigma}\right]\right. \\
& \left.+\sum_{i}\left[\Delta_{i}(\tau) \bar{\psi}_{i \uparrow}(\tau) \bar{\psi}_{i \downarrow}(\tau)+\text { H.c. }+\frac{\left|\Delta_{i}\right|^{2}}{|U|}\right]\right\} .
\end{aligned}
$$

The $\psi$ integral is now quadratic but at the cost of an additional integration over the fields $\Delta_{i}(\tau)$ and $\Delta_{i}^{*}(\tau)$. The "weight factor" for the $\Delta_{i}$ configurations can be determined by integrating out the $\psi, \bar{\psi}$, and using these weighted configurations one goes back and computes fermionic properties. Formally,

$$
\begin{aligned}
Z & =\int \mathcal{D} \Delta \mathcal{D} \Delta^{*} e^{-S_{2}\left[\Delta, \Delta^{*}\right]}, \\
S_{2} & =\ln \left[\operatorname{Det}\left[\mathcal{G}^{-1}-\Delta\right]\right]+\frac{\left|\Delta_{i}\right|^{2}}{|U|},
\end{aligned}
$$

where $\mathcal{G}$ is the electron Green's function in a $\left\{\Delta_{i}\right\}$ background.

The weight factor for an arbitrary space-time configuration $\Delta_{i}(\tau)$ involves computation of the fermionic determinant in that background. If we write the auxiliary field $\Delta_{i}(\tau)$ in terms of its Matsubara modes, as $\Delta_{i}\left(\Omega_{n}\right)$, then the various approximations can be readily recognized and compared.

(1) Quantum Monte Carlo retains the full " $i, \Omega_{n}$ " dependence of $\Delta$ computing $\ln \left[\operatorname{Det}\left[\mathcal{G}^{-1}-\Delta\right]\right]$ iteratively for importance sampling. The approach is valid at all $T$ but does not readily yield real frequency spectra.

(2) Mean-field theory (MFT) is time independent, neglects the phase fluctuations completely but can handle spatial inhomogeneity in amplitude of the pairing field. Thus $\Delta_{i}\left(i \Omega_{n}\right) \rightarrow$ $\Delta_{i}$. When the MF order parameter vanishes at high temperature, the theory trivializes.

(3) Our static path approximation (SPA) approach retains the full spatial dependence in $\Delta$ but keeps only the $\Omega_{n}=0$ mode, i.e., $\Delta_{i}\left(\Omega_{n}\right) \rightarrow \Delta_{i}$. It thus includes classical fluctuations of arbitrary magnitude but no quantum $\left(\Omega_{n} \neq 0\right)$ fluctuations. One may consider different temperature regimes. (i) $T=0$ : since classical fluctuations die off at $T=0$, SPA reduces to standard Bogoliubov-de Gennes (BdG) MFT. 
(ii) At $T \neq 0$ we consider not just the saddle point configuration but all configurations following the weight $e^{-S_{2}}$ above. These involve the classical amplitude and phase fluctuations of the order parameter, and the $\mathrm{BdG}$ equations are solved in all these configurations to compute the thermally averaged properties. This approach suppresses the order much quicker than in MFT. (iii) High $T$ : since the $\Omega_{n}=0$ mode dominates the exact partition function the SPA approach becomes exact as $T \rightarrow \infty$.

(4) DMFT: for completeness we mention that DMFT retains the full dynamics but keeps $\Delta$ at effectively one site, i.e., $\Delta_{i}\left(\Omega_{n}\right) \rightarrow \Delta\left(\Omega_{n}\right)$. This is exact when dimensionality $D \rightarrow \infty$.

\section{APPENDIX E: EFFECT OF QUANTUM FLUCTUATIONS}

The static path approximation used in this work gets progressively accurate with increasing temperature and is akin to the mean-field theory at the ground state. To that extent the ground-state phase diagram as reported in this manuscript is a mean-field estimate, where the quantum fluctuations are being neglected. We argue that the neglect of quantum fluctuations is a reasonable approximation for the present problem where the only gapless mode are the $X Y$-type low-energy excitations of the superconducting phase. Models with $X Y$ symmetry are well known to have long-range order in two dimensions and a BKT transition at finite temperature. The issue of fluctuations thus reduces to verifying how well the U(1) superconducting $T_{c}$ is captured by our approach as compared to that obtained via QMC. While QMC results for the present problem (straininduced SIT) are unavailable for comparison, the agreement between the results obtained through QMC and SPA for other systems (e.g., attractive Hubbard model on 2D square lattice [49]) suggests that the relevant fluctuations are suitably captured by our numerical technique.

Further, as we have discussed in our manuscript, in a recent work on attractive Hubbard model on the Lieb lattice, the results obtained via the mean-field theory (MFT) has been compared with those obtained via DMFT (which takes into account quantum fluctuations) [38]. It has been demonstrated that at the ground state, the results obtained by both the techniques are in fairly good agreement with each other, suggesting that the mean-field approach to the problem is good enough to capture the ground state. At high temperatures, MFT expectedly overestimates the thermal scales.

Based on the above discussion, we emphasize that MFT is a reasonably good approximation to capture the ground-state physics of the system discussed in this manuscript, and we do not expect any qualitative changes in the same through inclusion of quantum fluctuations.
[1] D. Meng, H. Guo, Z. Cui, C. Ma, J. Zhao, J. Lu, H. Xu, Z. Wang, X. Hu, Z. Fu, R. Peng, J. Guo, X. Zhai, G. J. Brown, R. Knize, and Y. Lu, Proc. Natl. Acad. Sci. USA 115, 2873 (2018).

[2] Z. Shao, Z.-G. Fu, S. Li, Y. Cao, Q. Bian, H. Sun, Z. Zhang, H. Gedeon, X. Zhang, L. Liu, Z. Cheng, F. Zheng, P. Zhang, and M. Pan, Nano Lett. 19, 5304 (2019).

[3] E.-M. Choi, A. Di Bernardo, B. Zhu, P. Lu, H. Alpern, K. H. L. Zhang, T. Shapira, J. Feighan, X. Sun, J. Robinson, Y. Paltiel, O. Millo, H. Wang, Q. Jia, and J. L. MacManus-Driscoll, Sci. Adv. 5, eaav5532 (2019).

[4] J. Engelmann, V. Grinenko, P. Chekhonin, W. Skrotzki, D. V. Efremov, S. Oswald, K. Iida, R. Hühne, J. Hänisch, M. Hoffmann, F. Kurth, L. Schultz, and B. Holzapfel, Nat. Commun. 4, 2877 (2013).

[5] W. Liang, Z. Zhuo, Y. Ji, C. Lu, M. Gao, H. Yang, C. Chen, F. Pan, and Y. Lin, npj Quantum Mater. 4, 39 (2019).

[6] Z. H. Ni, T. Yu, Y. H. Lu, Y. Y. Wang, Y. P. Feng, and Z. X. Shen, ACS Nano 2, 2301 (2008).

[7] C. Lee, X. Wei, J. W. Kysar, and J. Hone, Science 321, 385 (2008).

[8] G. G. Naumis, S. Barraza-Lopez, M. Oliva-Leyva, and H. Terrones, Rep. Prog. Phys. 80, 096501 (2017).

[9] D. Akinwande, N. Petrone, and J. Hone, Nat. Commun. 5, 5678 (2014).

[10] B. Amorim, A. Cortijo, F. de Juan, A. Grushin, F. Guinea, A. Gutierrez-Rubio, H. Ochoa, V. Parente, R. Roldan, P. San-Jose, J. Schiefele, M. Sturla, and M. Vozmediano, Phys. Rep. 617, 1 (2016).

[11] F. Guinea, M. I. Katsnelson, and A. K. Geim, Nat. Phys. 6, 30 (2010).

[12] A. C. McRae, G. Wei, and A. R. Champagne, Phys. Rev. Appl. 11, 054019 (2019).
[13] D. Leykam, A. Andreanov, and S. Flach, Adv. Phys.: X 3, 1473052 (2018).

[14] O. Derzhko, J. Richter, and M. Maksymenko, Int. J. Mod. Phys. B 29, 1530007 (2015).

[15] S. A. Parameswaran, R. Roy, and S. L. Sondhi, C. R. Phys. 14, 816 (2013).

[16] E. J. Bergholtz and Z. Liu, Int. J. Mod. Phys. B 27, 1330017 (2013).

[17] B. Sutherland, Phys. Rev. B 34, 5208 (1986).

[18] E. H. Lieb, Phys. Rev. Lett. 62, 1201 (1989).

[19] A. Mielke, J. Phys. A 24, L73 (1991).

[20] H. Tasaki, Phys. Rev. Lett. 69, 1608 (1992).

[21] N. Shima and H. Aoki, Phys. Rev. Lett. 71, 4389 (1993).

[22] C. Weeks and M. Franz, Phys. Rev. B 82, 085310 (2010).

[23] S. Taie, H. Ozawa, T. Ichinose, T. Nishio, S. Nakajima, and Y. Takahashi, Sci. Adv. 1, e1500854 (2015).

[24] H. Ozawa, S. Taie, T. Ichinose, and Y. Takahashi, Phys. Rev. Lett. 118, 175301 (2017).

[25] R. Drost, T. Ojanen, A. Harju, and P. Liljeroth, Nat. Phys. 13, 668 (2017).

[26] M. R. Slot, T. S. Gardenier, P. H. Jacobse, G. C. P. van Miert, S. N. Kempkes, S. J. M. Zevenhuizen, C. M. Smith, D. Vanmaekelbergh, and I. Swart, Nat. Phys. 13, 672 (2017).

[27] W.-X. Qiu, S. Li, J.-H. Gao, Y. Zhou, and F.-C. Zhang, Phys. Rev. B 94, 241409(R) (2016).

[28] R. A. Vicencio, C. Cantillano, L. Morales-Inostroza, B. Real, C. Mejía-Cortés, S. Weimann, A. Szameit, and M. I. Molina, Phys. Rev. Lett. 114, 245503 (2015).

[29] S. Xia, Y. Hu, D. Song, Y. Zong, L. Tang, and Z. Chen, Opt. Lett. 41, 1435 (2016).

[30] S. Mukherjee and R. R. Thomson, Opt. Lett. 40, 5443 (2015). 
[31] R. Chen, D.-H. Xu, and B. Zhou, Phys. Rev. B 96, 205304 (2017).

[32] A. Zhao and S.-Q. Shen, Phys. Rev. B 85, 085209 (2012).

[33] S. Takayoshi, H. Katsura, N. Watanabe, and H. Aoki, Phys. Rev. A 88, 063613 (2013).

[34] W.-F. Tsai, C. Fang, H. Yao, and J. Hu, New J. Phys. 17, 055016 (2015).

[35] M. Metcalf, G.-W. Chern, M. D. Ventra, and C.-C. Chien, J. Phys. B 49, 075301 (2016).

[36] M. Bercx, J. S. Hofmann, F. F. Assaad, and T. C. Lang, Phys. Rev. B 95, 035108 (2017).

[37] M. Tylutki and P. Törmä, Phys. Rev. B 98, 094513 (2018).

[38] A. Julku, S. Peotta, T. I. Vanhala, D.-H. Kim, and P. Törmä, Phys. Rev. Lett. 117, 045303 (2016).

[39] J. Gouveia and R. Dias, J. Magn. Magn. Mater. 382, 312 (2015).

[40] J. Gouveia and R. Dias, J. Magn. Magn. Mater. 405, 292 (2016).

[41] P. Kumar, T. I. Vanhala, and P. Törmä, Phys. Rev. B 96, 245127 (2017).

[42] K. Noda, K. Inaba, and M. Yamashita, Phys. Rev. A 91, 063610 (2015).

[43] J. Faúndez, T. N. Jorge, and L. Craco, Phys. Rev. B 97, 115149 (2018).

[44] N. C. Costa, T. Mendes-Santos, T. Paiva, R. R. dos Santos, and R. T. Scalettar, Phys. Rev. B 94, 155107 (2016).

[45] S. Peotta and P. Törmä, Nat. Commun. 6, 8944 (2015).

[46] H. Aoki, M. Ando, and H. Matsumura, Phys. Rev. B 54, R17296 (1996).

[47] W. E. Evenson, J. R. Schrieffer, and S. Q. Wang, J. Appl. Phys. 41, 1199 (1970).

[48] Y. Dubi, Y. Meir, and Y. Avishai, Nature (London) 449, 876 (2007).

[49] S. Tarat and P. Majumdar, Eur. Phys. J. B 88, 68 (2015).

[50] M. Karmakar and P. Majumdar, Phys. Rev. A 93, 053609 (2016).

[51] N. Swain and P. Majumdar, Europhys. Lett. 119, 17004 (2017).

[52] M. Karmakar, Phys. Rev. A 97, 033617 (2018).

[53] A. Dutta, A. Joshi, K. Sengupta, and P. Majumdar, Phys. Rev. B 99, 195126 (2019).

[54] M. G. Ries, A. N. Wenz, G. Zürn, L. Bayha, I. Boettcher, D. Kedar, P. A. Murthy, M. Neidig, T. Lompe, and S. Jochim, Phys. Rev. Lett. 114, 230401 (2015).

[55] V. I. Iglovikov, F. Hébert, B. Grémaud, G. G. Batrouni, and R. T. Scalettar, Phys. Rev. B 90, 094506 (2014).

[56] Y. L. Loh, M. Randeria, N. Trivedi, C.-C. Chang, and R. Scalettar, Phys. Rev. X 6, 021029 (2016).

[57] M. Randeria and E. Taylor, Annu. Rev. Condens. Matter Phys. 5, 209 (2014).

[58] A. Ghosal, M. Randeria, and N. Trivedi, Phys. Rev. Lett. 81, 3940 (1998).
[59] A. Ghosal, M. Randeria, and N. Trivedi, Phys. Rev. B 65, 014501 (2001).

[60] K. Bouadim, Y. L. Loh, M. Randeria, and N. Trivedi, Nat. Phys. 7, 884 (2011).

[61] N. Trivedi, R. T. Scalettar, and M. Randeria, Phys. Rev. B 54, R3756 (1996).

[62] R. T. Scalettar, N. Trivedi, and C. Huscroft, Phys. Rev. B 59, 4364 (1999).

[63] M. Swanson, Y. L. Loh, M. Randeria, and N. Trivedi, Phys. Rev. X 4, 021007 (2014).

[64] E. Jin, M. Asada, Q. Xu, S. Dalapati, M. A. Addicoat, M. A. Brady, H. Xu, T. Nakamura, T. Heine, Q. Chen, and D. Jiang, Science 357, 673 (2017).

[65] W. Jiang, H. Huang, and F. Liu, Nat. Commun. 10, 2207 (2019).

[66] C. Chin, M. Bartenstein, A. Altmeyer, S. Riedl, S. Jochim, J. H. Denschlag, and R. Grimm, Science 305, 1128 (2004).

[67] J. P. Gaebler, J. T. Stewart, T. E. Drake, D. S. Jin, A. Perali, P. Pieri, and G. C. Strinati, Nat. Phys. 6, 569 (2010).

[68] Y.-i. Shin, C. H. Schunck, A. Schirotzek, and W. Ketterle, Nature (London) 451, 689 (2008).

[69] C. H. Schunck, Y. Shin, A. Schirotzek, M. W. Zwierlein, and W. Ketterle, Science 316, 867 (2007).

[70] P. Törmä and P. Zoller, Phys. Rev. Lett. 85, 487 (2000).

[71] G. M. Bruun, P. Törmä, M. Rodriguez, and P. Zoller, Phys. Rev. A 64, 033609 (2001).

[72] Y. He, Q. Chen, and K. Levin, Phys. Rev. A 72, 011602(R) (2005).

[73] J. Kinnunen, M. Rodríguez, and P. Törmä, Science 305, 1131 (2004).

[74] C. A. Regal and D. S. Jin, Phys. Rev. Lett. 90, 230404 (2003).

[75] S. Gupta, Z. Hadzibabic, M. W. Zwierlein, C. A. Stan, K. Dieckmann, C. H. Schunck, E. G. M. van Kempen, B. J. Verhaar, and W. Ketterle, Science 300, 1723 (2003).

[76] M. W. Zwierlein, Z. Hadzibabic, S. Gupta, and W. Ketterle, Phys. Rev. Lett. 91, 250404 (2003).

[77] A. Perali, P. Pieri, and G. C. Strinati, Phys. Rev. Lett. 100, 010402 (2008).

[78] P. Pieri, A. Perali, and G. C. Strinati, Nat. Phys. 5, 736 (2009).

[79] P. Pieri, A. Perali, G. C. Strinati, S. Riedl, M. J. Wright, A. Altmeyer, C. Kohstall, E. R. Sánchez Guajardo, J. Hecker Denschlag, and R. Grimm, Phys. Rev. A 84, 011608(R) (2011).

[80] A. Schirotzek, Y.-i. Shin, C. H. Schunck, and W. Ketterle, Phys. Rev. Lett. 101, 140403 (2008).

[81] J. T. Stewart, J. P. Gaebler, and D. S. Jin, Nature (London) 454, 744 (2008).

[82] J. Kinnunen, M. Rodríguez, and P. Törmä, Phys. Rev. Lett. 92, 230403 (2004). 